\title{
Analysis of Packet Interference and Aggregated Throughput in a Cluster of Bluetooth Piconets Under Different Traffic Conditions
}

\author{
Kshirasagar Naik, Member, IEEE, David S. L. Wei, Member, IEEE, Yu T. Su, Member, IEEE, and \\ Norio Shiratori, Fellow, IEEE
}

\begin{abstract}
In a Bluetooth piconet, the Master essentially controls the channel. Due to an absence of coordination between independent Masters while accessing the wireless medium, devices will encounter high packet interference if several piconets are simultaneously operating in the same area. Since even a headset and a mobile phone can be connected with a Bluetooth link forming a piconet, it may not be unusual to find tens of independent piconets in crowded places like airports, international conferences, shopping malls, and so on. Study of packet interference is important because interference affects the throughput of a piconet. Motivated by the fact that applications will benefit, in terms of higher available data rate in one direction, by using multiple-slot packets in an asymmetric manner, in this paper, we present an analytical model of packet interference in a cluster of piconets using multiple-slot packets. Also, considering that all the portable devices can have a Bluetooth interface and people are highly mobile these days, it will not be uncommon to find a cluster of piconets of both the 79-hop and the 23-hop types in the same area. We then present an analytical model of interference of multiple-slot packets in a heterogeneous cluster of Bluetooth piconets. By a heterogeneous cluster we mean some piconets are of the 23-hop type and the rest are of 79-hop type. We show how the aggregate throughput in a cluster of piconets degrade under various traffic scenarios, such as 1-slot, 3-slot, and 5-slot packets in symmetric and asymmetric modes in synchronous and asynchronous conditions of Master clocks. Our analytic model is based on the idea of probabilistic graphs, where a node denotes a piconet and an edge denotes the probability of interference between two nodes. Though the 23-hop system has been phased out, our work gives a general approach to model packet interference in multiple, frequency-hopping systems that need not be Bluetooth systems.
\end{abstract}

Index Terms-Bluetooth technology, packet interference, frequency-hopping system, probabilistic graph.

\section{INTRODUCTION}

I $\mathrm{N}$ A Bluetooth piconet, the Master essentially controls the channel [4]. Due to an absence of coordination between independent Masters while accessing the wireless medium, de-

Manuscript received February 1, 2004; revised January 20, 2005. This paper was presented in part at ICC 2004, Paris, France.

K. Naik is with the Department of Electrical and Computer Engineering, University of Waterloo, Waterloo, ON N2L 3G1, Canada (e-mail: knaik@swen. uwaterloo.ca).

D. S. L. Wei is with the Department of Computer and Information Sciences, Fordham University, Bronx, NY 10458 USA (e-mail: wei@ dsm.fordham.edu).

Y. T. Su is with the Department of Communications Engineering, National Chiao Tung University, Hsinchu 300, Taiwan (e-mail: ytsu@ mail.nctu.edu.tw).

N. Shiratori is with the Research Institute of Electrical Communication, Tohoku University, Sendai 980-8577, Japan (e-mail: norio@shiratori. riec.ac.jp).

Digital Object Identifier 10.1109/JSAC.2005.845629 vices will encounter high packet interference if several piconets are simultaneously operating in the same area. Since even a headset and a mobile phone can be connected with a Bluetooth link forming a piconet, it may not be unusual to find tens of independent piconets in crowded places like airports, international conferences, shopping malls, and so on. Study of packet interference gains importance because it affects throughput of a piconet. Therefore, it has been attracting the attentions of several researchers [1]-[3], [5]-[9].

El-Hoiydi [1] has given a probabilistic model of interference of single-slot packets in a homogeneous cluster of piconets, i.e., all piconets are either of 79-hop type or of 23-hop type. Considering that all the portable devices can have a Bluetooth interface and people are highly mobile these days, it will not be uncommon to find a cluster of piconets of both the 79-hop and the 23-hop types in the same area. Naik et al. [7] have given a generalized model of interference of single-slot packets in a heterogeneous cluster of piconets. A heterogeneous cluster means all the devices in some piconets are of 79-hop type and all the devices in the rest of the piconets are of 23-hop types. (The reader may note that in our present study, a heterogeneous cluster does not include a piconet where the Master is of the 79-hop (or, 23-hop) type and a Slave is of the 23-hop (or, 79-hop) type.) The model of Naik et al. [7] uses the idea of a probabilistic graph.

In Table I, we have summarized the data rates obtained at the baseband level by using different types of packets. Packet types such as DM1 and DH1 occupy a single slot, and deliver a maximum data rate of $172.8 \mathrm{Kbps}$ in both the directions-forward or reverse. Data traffic between the Master and a Slave in a piconet is said to be symmetric if both the devices get equal number of slots to transmit their data. To obtain higher data rates, packets occupying multiple slots, such as three and five, must be used. Moreover, data traffic is generally asymmetric in applications such as Internet browsing.

Therefore, one end of a network-based application will receive a higher bandwidth by using multiple-slot packets in an asymmetric manner. In this way, a user can obtain a data rate of up to $723.2 \mathrm{~kb} / \mathrm{s}$ while downloading data from the Internet, and a rate of $57.6 \mathrm{~kb} / \mathrm{s}$ while uploading. Therefore, it is highly likely that an application will prefer multiple-slot packets for data delivery. For voice, applications will use single-slot packets HV1, $\mathrm{HV} 2$, or HV3 to achieve a fixed rate of $64.0 \mathrm{~kb} / \mathrm{s}$.

Motivated by the above observation, we present a model of packet interference in a cluster of piconets using multiple-slot packets. Once again, our model is based on the idea of a 
TABLE I

DATA RATES OFFERED BY DIFFERENT TYPES OF PACKETS

\begin{tabular}{c|c|c|c|c|c|c}
\hline Type & $\begin{array}{c}\text { User payload } \\
\text { in bytes }\end{array}$ & $\begin{array}{c}\text { Forward } \\
\text { Error } \\
\text { Correction }\end{array}$ & $\begin{array}{c}\text { \# of slots } \\
\text { occupied }\end{array}$ & $\begin{array}{c}\text { Symmetric } \\
\text { Max. rate (Kbps) }\end{array}$ & \multicolumn{2}{c}{$\begin{array}{c}\text { Asymmetric } \\
\text { Max. rate (Kbps) } \\
\text { Forward }\end{array}$} \\
Reverse \\
\hline DM1 & $0-17$ & $2 / 3$ & 1 & 108.8 & 108.8 & 108.8 \\
\hline DH1 & $0-27$ & no & 1 & 172.8 & 172.8 & 172.8 \\
\hline DM3 & $0-121$ & $2 / 3$ & 3 & 258.1 & 387.2 & 54.4 \\
\hline DH3 & $0-183$ & no & 3 & 390.4 & 585.6 & 86.4 \\
\hline DM5 & $0-224$ & $2 / 3$ & 5 & 286.7 & 477.8 & 36.3 \\
\hline DH5 & $0-339$ & no & 5 & 433.9 & 723.2 & 57.6 \\
\hline
\end{tabular}

probabilistic graph explained in Section II. Our model considers the following aspects of data transmission.

- Symmetric and asymmetric traffic.

- A cluster of either homogeneous or heterogeneous piconets using a mix of 1-slot, 3-slot, and 5-slot packets.

- Synchrony and asynchrony between packets transmitted in different piconets.

While using multiple-slot packets such as DM3, DH3, DM5, and DH5, one will have to decide whether to use these packets in a symmetric or an asymmetric manner. Symmetric traffic using 3-slot packets and 5-slot packets are explained as follows. (The reader may note that traffic due to 1 -slot packet transmission is inherently symmetric.)

- Symmetric 3-slot packets: In a block of six consecutive slots, the Master transmits a 3-slot packet to a Slave, and the Slave transmits a 3-slot packet to the Master.

- Asymmetric 3-slot packets: In a block of four consecutive slots, the Master transmits a 3-slot packet to a Slave, and the Slave transmits a 1-slot packet to the Master.

- Symmetric 5-slot packets: In a block of ten consecutive slots, the Master transmits a 5-slot packet to a Slave, and the Slave transmits a 5-slot packet to the Master.

- Asymmetric 5-slot packets: In a block of six consecutive slots, the Master transmits a 5-slot packet to a Slave, and the Slave transmits a 1-slot packet to the Master.

For our study, we need to define the idea of synchronization of packet transmission between two piconets transmitting packets of different lengths (in number of slots). It is not hard to see that it is not possible to synchronize every Tx slot under the traffic with multiple-slot packets. However, one can easily observe that if two piconets are both in the symmetric mode or both in the asymmetric mode, and they start transmission of a Tx slot or an Rx slot at the same time at some instance, then they will always start transmission of a Tx slot at the same time for every fixed period of time. To be able to compare packet loss between the synchronized operation and unsynchronized operation of two piconets, we assume that if two piconets start transmission of a Tx slot or an Rx slot at the same time at some instances, they are treated as operating synchronously. If two piconets are not synchronized as defined above, they are said to be asynchronous. There is a need to make this distinction because it affects packet interference. For example, the probability of interference between two asynchronous piconets transmitting 1-slot packets is twice of that between two synchronous piconets [7].

We compute packet interference and aggregated throughput in a cluster of piconets as follows. We model packet interference as a probabilistic graph, where a node represents a piconet. The weight of an edge between two nodes in the probabilistic graph denotes the probability of packet interference between the two nodes in a given traffic condition. We show how to compute these probability quantities for various types of data traffic. The average number of nodes without an incident edge represents the average number of packets at a given moment not interfered. Thus, the amount of interference is computed by subtracting the said quantity from the number of piconets. We use the average number of piconets whose packets are not interfered at a given moment and the amount of data carried by those packets to compute the aggregated throughput. Finally, we show how packet interference and aggregated throughput change as a function of traffic type and the number of piconets in a cluster.

The paper has been organized as follows. The idea of modeling packet interference as a probabilistic graph is explained in Section II. Interference among homogeneous piconets using multiple-slot packets is studied in Section III. Interference among heterogeneous piconets using multiple-slot packets is studied in Section IV. Some concluding remarks are given in Section V.

\section{Probabilistic GraphS IN Modeling InTERFERENCE}

In an earlier work [7], we have introduced the concept of a probabilistic graph to model single-slot packet interference in a heterogeneous cluster of piconets. Thus, the result reported by El-Hoiydi [1] to model packet interference in a homogeneous cluster of piconets becomes a special case of our work reported in [7]. In this paper, we use the same concept of a probabilistic graph to model interference in a cluster of piconets with a variety of traffic types, such as symmetric and asymmetric and synchronous and asynchronous, with multislot packets. In the rest of this section, we summarize the concept of probabilistic graphs in modeling packet interference.

Let $G=(V, E)$ be a probabilistic graph, where $V$ is a set of nodes and $E$ is a set of edges of $G$. Let $N=|V|$ denote the number of nodes in $G$. Each node in $G$ represents a piconet. The weight of an edge between two nodes $v_{i}$ and $v_{j}$ is denoted by $p$. The quantity $p$ represents the probability of a packet in one piconet, say, $v_{i}$, being interfered by a packet in another piconet, say, $v_{j}$. A pair of packets transmitted in two piconets are said to interfere with each other if the packets are transmitted on the same frequency and the two packets overlap. Let $X_{N}$ be a random variable representing the number of nodes in $G$ with no incident edges. Thus, $X_{N}$ is a measure of the number of packets not being interfered. The expected value of $X_{N}$, denoted by $E\left(X_{N}\right)$, gives the average number of packets that are not interfered. The quantity $N-E\left(X_{N}\right)$ represents the expected number of packets that are interfered. 
Therefore, the two key elements in computing packet interference in a cluster of piconets tansmitting multiple-slot packets are as follows:

- identifying values of $p$;

- giving an expression for $E\left(X_{N}\right)$.

The quantity $p$ depends on three factors, namely, the following:

- the hop type of each piconet: 23-hop or 79-hop;

- whether or not two piconets are synchronized as defined before;

- packet length in number of slots.

Giving an expression for the quantity $E\left(X_{N}\right)$ for a cluster of $N$ piconets with no pattern of the above three factors is a difficult task. Therefore, we will consider the following scenarios.

- First, we will assume that all the piconets are homogeneous, i.e., either of 79-hop type or of 23-hop type. Let $s$ represent the number of frequencies available in a piconet. In the 23-hop and 79-hop systems $s=23$ and $s=79$, respectively.

- Next, we consider the heterogeneous case.

- Finally, we consider symmetric and asymmetric traffic separately.

In the following sections, we take up these cases one by one.

\section{INTERFERENCE MODEL OF HOMOGENEOUS SYSTEM}

\section{A. Computing $p_{i j}$ S Under Symmetric Traffic}

Data traffic in a piconet is said to be symmetric if both the Master and a Slave transmit at the same rate. This implies that they equally share the channel slots. Let $p_{i j}$ be the interference probability between a piconet transmitting $i$-slot packets and a piconet transmitting $j$-slot packets. The variables $i$ and $j$ can take on discrete values from the set $\{1,3,5\}$. To simplify the discussion, in the calculation of $p_{i j} \mathrm{~s}$, we assume that the packet to slot ratio is one, i.e., the duration occupied by the $i$-slot packet is exactly the same as the total length of $i \operatorname{slot}(\mathrm{s})$ for $i=1,3$, and 5. Also, be informed that the $p_{i j} \mathrm{~s}$ in [7] are computed under the assumption that two consecutive $\mathrm{Tx}$ and $\mathrm{Rx}$ slots will not use the same hop frequency according to the Bluetooth specification [4], which is right for a piconet using single-slot packets. But in a cluster of piconets with multiple-slot packets, when computing $p_{31}$ or $p_{51}$, as an example, it is assumed that in any run of three or five consecutive slots in a 1-slot piconet a hop frequency is not repeated. ${ }^{1}$

1) Two Piconets Are Synchronized: For discussion convenience, let $\operatorname{Tx}(1), \operatorname{Tx}(3)$, and $\operatorname{Tx}(5)$ be the $\operatorname{Tx}$ slot of 1-slot, 3 -slot, and 5-slot, respectively, and let $\operatorname{Rx}(1), \operatorname{Rx}(3)$, and $\operatorname{Rx}(5)$ be the Rx slot of 1-slot, 3-slot, and 5-slot, respectively. Let $s$ be the number of frequencies (hops) available in a piconet (for example, in the 79-hop system $s=79$ ), we validate the following lemma.

Lemma 3.1: Under symmetric traffic, if the two Masters M1 and $\mathrm{M} 2$ are synchronized, we have $p_{11}=1 / s, p_{33}=1 / s$, $p_{55}=1 / s, p_{13}=1 / s, p_{31}=3 / s, p_{15}=1 / s, p_{51}=5 / s$, $p_{35}=7 / 5 s$, and $p_{53}=7 / 3 s$.

\footnotetext{
${ }^{1}$ In reality, the probability that a hop frequency is repeated in any run of three or five consecutive slots is extremely low. Thus, to simplify the discussion, we assume that it is 0 .
}

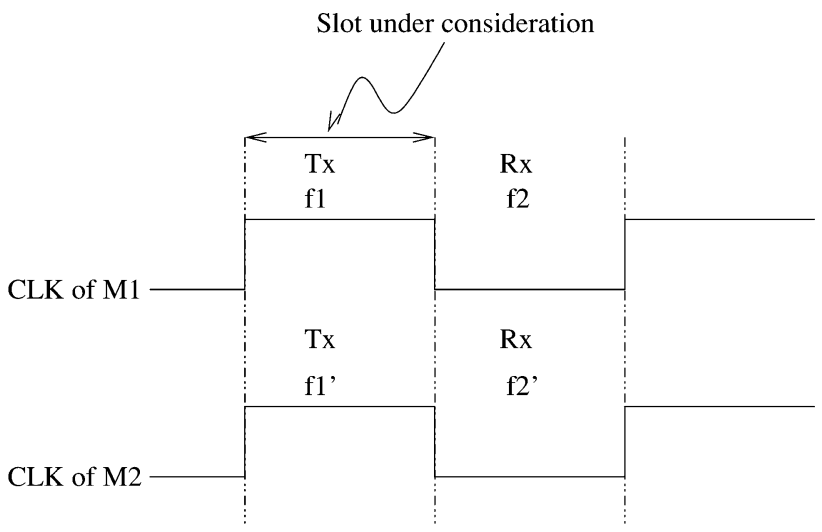

Fig. 1. Packet interference.

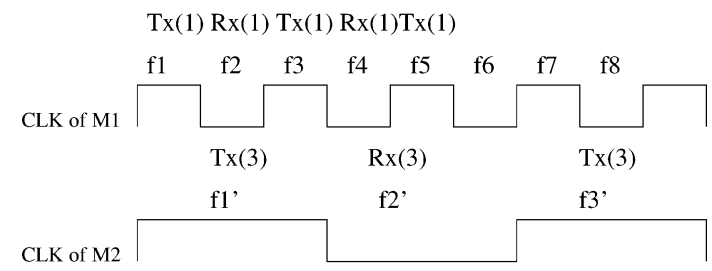

Fig. 2. Interference between two synchronized piconets (1- and 3-slot packets).

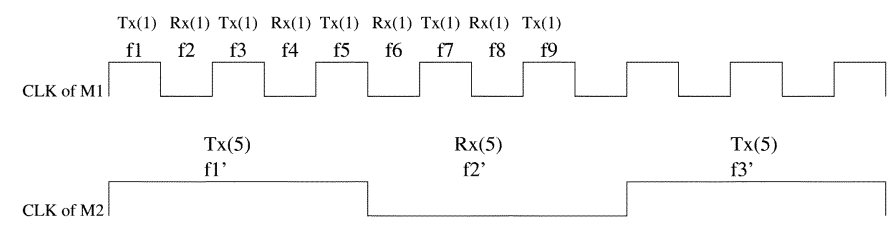

Fig. 3. Interference between two synchronized piconets (1- and 5-slot packets).

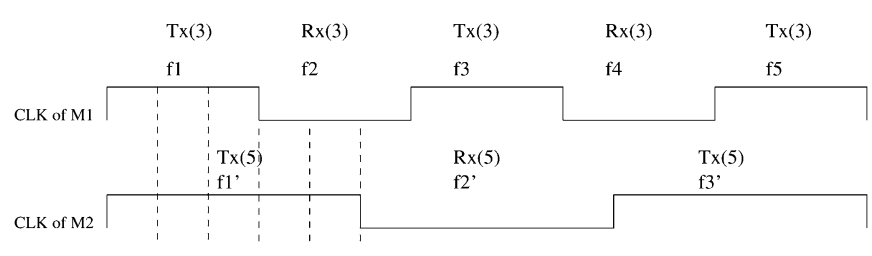

Fig. 4. Interference between two synchronized piconets (3- and 5-slot packets).

Proof: Using Fig. 1, equations $p_{11}=1 / s, p_{33}=1 / s$, $p_{55}=1 / s$, can be easily verified. When using Fig. 1 for verifying $p_{33}=1 / s$ and $p_{55}=1 / s$, one must view each slot in the figure as a 3 -slot and 5-slot packet, respectively. Also, using Fig. 2 Equations $p_{31}=3 / s$ and $p_{13}=1 / s$ can be verified, and using Fig. 3, equations $p_{51}=5 / s$ and $p_{15}=1 / s$ can also be easily verified. To validate $p_{53}=7 / 3 s$, we use Fig. 4 as follows. Using Fig. 4, one can see that a Tx(5) of M2 overlaps with a $\operatorname{Tx}(3)$ and an $\operatorname{Rx}(3)$ of $M 1$, in which the interference probability is $2 / \mathrm{s}$, and the following $\operatorname{Rx}(5)$ overlaps with an $\operatorname{Rx}(3)$, a $\mathrm{Tx}(3)$, and an $\operatorname{Rx}(3)$ of $M 1$, in which the interference probability is $3 / \mathrm{s}$, and then the following $\operatorname{Tx}(5)$ overlaps with an $\operatorname{Rx}(3)$ and a $\operatorname{Tx}(3)$ of M1, in which the packet interference probability is $2 / s$. Then, the next $\operatorname{Rx}(5)$ of M2 is synchronized with the next 


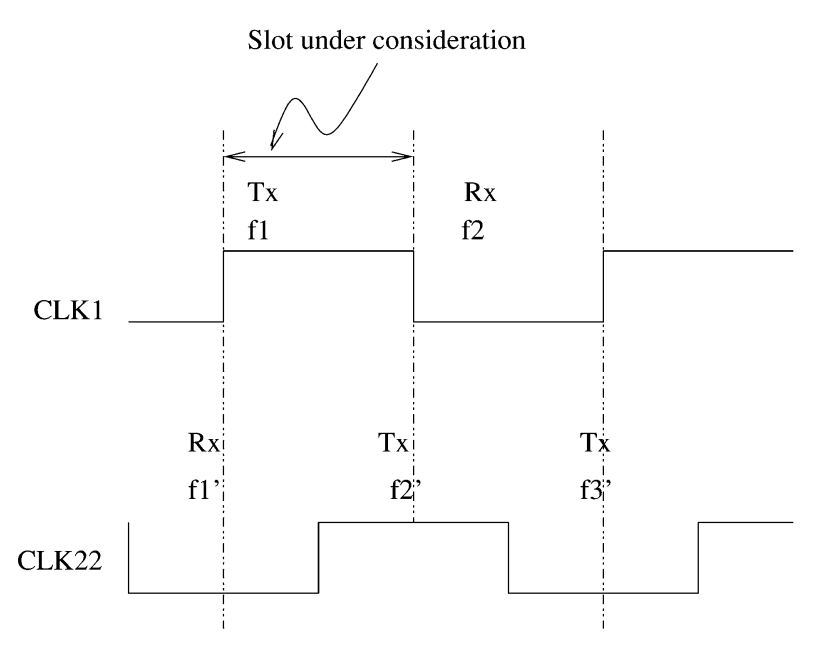

Fig. 5. Packet interference.

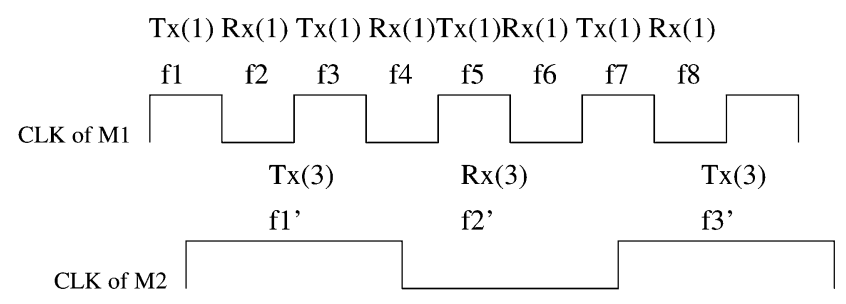

Fig. 6. Interference between two asynchronized piconets (1- and 3-slot packets).

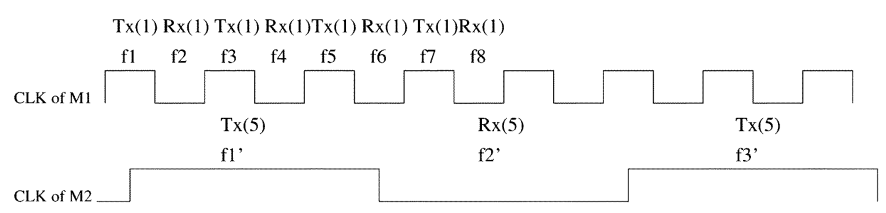

Fig. 7. Interference between two asynchronized piconets (1- and 5-slot packets).

$\operatorname{Rx}(3)$ of M1 again, which means that the above stated situtatiom will be repeated again for the next three 5-slot packets of M2 and the next five 3-slot packets of M1. We, thus, conclude that $p_{53}=(2 / s+3 / s+2 / s) / 3=7 / 3 s$.

Using Fig. 4 , in a similar way, one can also show that $p_{35}=$ $(1 / s+2 / s+1 / s+2 / s+1 / s) / 5=7 / 5 s$.

2) Two Piconets are Asynchronous:

Lemma 3.2: Under symmetric traffic, if the two Masters M1 and M2 operate asynchronously, we have $p_{11}=2 / s, p_{33}=$ $2 / s, p_{55}=2 / s, p_{13}=4 / 3 s, p_{31}=4 / s, p_{15}=6 / 5 s, p_{51}=$ $6 / s, p_{35}=8 / 5 s$, and $p_{53}=8 / 3 s$.

Proof: Using Fig. 5, one can easily verify that $p_{11}=2 / s$, $p_{33}=2 / s, p_{55}=2 / s$. When using Fig. 5 for verifying $p_{33}=$ $2 / s$ and $p_{55}=2 / s$, one must view each slot in the figure as a 3 -slot and 5-slot packet, respectively. Also, using Figs. 6 and 7, it is not hard to validate $p_{31}=4 / s$ and $p_{51}=6 / s$, respectively, and validate $p_{13}=(2 / s+1 / s+1 / s) / 3=4 / 3 s$ and $p_{15}=$ $(2 / s+1 / s+1 / s+1 / s+1 / s) / 5=6 / 5 s$, respectively. Now, using Fig. 8 , we can verify that $p_{53}=8 / 3 s$ in three cases.

Case 1) $(0<$ shift $<1$-slot $)$ : In this case, a $\operatorname{Tx}(5)$ of M2 overlaps with a $\operatorname{Tx}(3)$ and an $\operatorname{Rx}(3)$ of M1, in which the interference probability is $2 / \mathrm{s}$, and the following $\operatorname{Rx}(5)$ overlaps with an $\operatorname{Rx}(3)$, a $\operatorname{Tx}(3)$,

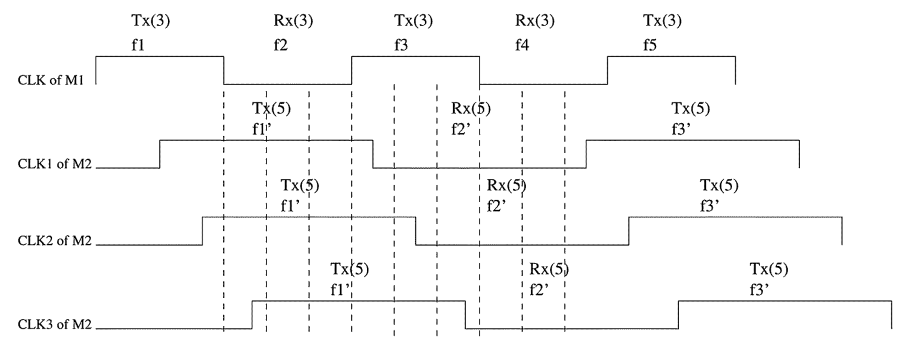

Fig. 8. Interference between two symmetric, asynchronized piconets (3- and 5-slot packets).

and an $\operatorname{Rx}(3)$ of M1, in which the interference probability is $3 / \mathrm{s}$, and then the following $\operatorname{Tx}(5)$ overlaps with an $\operatorname{Rx}(3)$, a $\operatorname{Tx}(3)$, and an $\operatorname{Rx}(3)$ of $M 1$, in which the packet interference probability is $3 / \mathrm{s}$. Then, the same situation will be repeated again for the next three 5-slot packets of M2. Thus, the average interference probability for this case is $(2 / s+$ $3 / s+3 / s) / 3=8 / 3 s$.

Case 2) (1-slot $<$ shift $<2$-slot): In this case, a $\operatorname{Tx}(5)$ of M2 overlaps with a $\operatorname{Tx}(3)$, an $\operatorname{Rx}(3)$, and a $\operatorname{Tx}(3)$ of $\mathrm{M} 1$, in which the interference probability is $3 / \mathrm{s}$, and the following $\operatorname{Rx}(5)$ overlaps with a $\operatorname{Tx}(3)$ and an $\operatorname{Rx}(3)$ of M1, in which the interference probability is $2 / \mathrm{s}$, and then the following $\operatorname{Tx}(5)$ overlaps with an $\operatorname{Rx}(3)$, a $\operatorname{Tx}(3)$, and an $\operatorname{Rx}(3)$ of $M 1$, in which the packet interference probability is $3 / \mathrm{s}$. Then, the same situation will be repeated again for the next three 5-slot packets of M2. Thus, the average interference probability for this case is $(3 / s+2 / s+$ $3 / s) / 3=8 / 3 s$.

Case 3) (2-slot $<$ shift $<3$-slot): In this case, a $\operatorname{Tx}(5)$ of M2 overlaps with a $\operatorname{Tx}(3)$, an $\operatorname{Rx}(3)$, and a $\operatorname{Tx}(3)$ of M1, in which the interference probability is $3 / \mathrm{s}$, and the following $\operatorname{Rx}(5)$ of M2 overlaps with a $\operatorname{Tx}(3)$, an $\operatorname{Rx}(3)$, and a $\operatorname{Tx}(3)$ of $M 1$, in which the interference probability is $3 / \mathrm{s}$, and then the next $\operatorname{Tx}(5)$ of M2 overlaps with a $\operatorname{Tx}(3)$ and an $\operatorname{Rx}(3)$ of M1, in which the packet interference probability is $2 / \mathrm{s}$. Then, the same situation will be repeated again for the next three 5-slot packets of M2. Thus, the average interference probability for this case is $(3 / s+3 / s+2 / s) / 3=8 / 3 s$. We, thus, conclude that $p_{53}=1 / 3 \times 8 / 3 s+1 / 3 \times 8 / 3 s+1 / 3 \times 8 / 3 s=$ $8 / 3 s$.

In a similar way and using the same figure, one can also verify that the interference probability of each of the three cases for $p_{35}$ is $8 / 5 s$, and we thus have $p_{35}=8 / 5 s$.

\section{B. Computing $p_{i j} s$ Under Asymmetric Traffic}

Again, let $p_{i j}$ be the interference probability between a piconet transmitting $i$-slot packets and a piconet transmitting $j$-slot packets. Be reminded that under the scenario of asymmetric traffic, a piconet of $i$-slot type transmits $i$-slot packets from the Master to a Slave (downstream traffic), and transmits 1-slot packet (upstream traffic) from a Slave to the Master, or vice versa. Since we are interested in the packet loss of a 


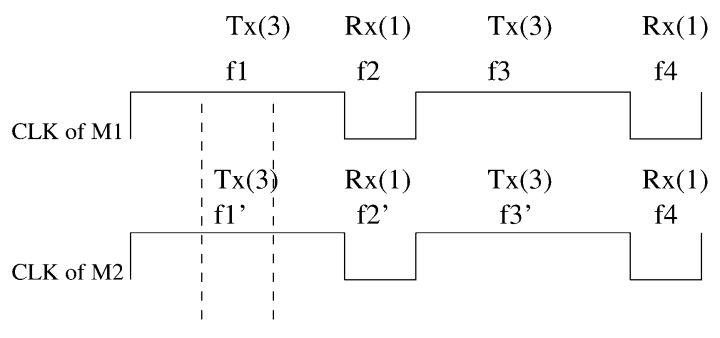

Fig. 9. Interference between two asymmetric, synchronized piconets (3-slot packets).

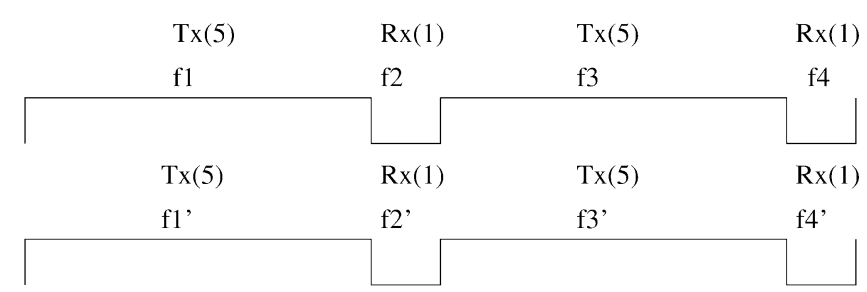

Fig. 10. Interference between two asymmetric, synchronized piconets (5-slot packets).

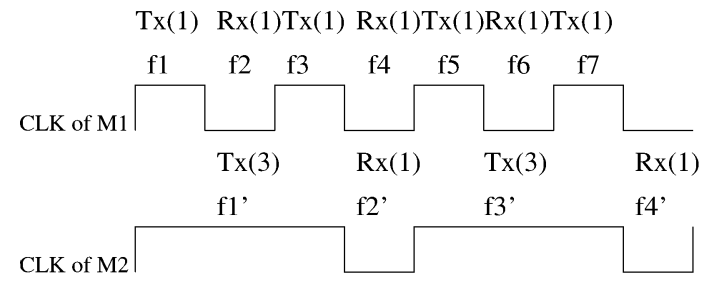

Fig. 11. Interference between two asymmetric, synchronized piconets (1- and 3-slot packets).

piconet rather than the packet loss of each individual Bluetooth device within the piconet, the upstream and downstream traffic will be treated together.

1) Two Piconets Are Synchronized: When the two piconets operate "synchronously," we have the following lemma.

Lemma 3.3: Under asymmetric traffic, if the two Masters M1 and M2 operate synchronously, we have $p_{11}=1 / s, p_{33}=1 / s$, $p_{55}=1 / s, p_{13}=1 / s, p_{31}=2 / s, p_{15}=1 / s, p_{51}=3 / s$, $p_{35}=4 / 3 s$, and $p_{53}=2 / s$.

Proof: Using Figs. 1, 9, and 10, one can easily see that $p_{11}=1 / s, p_{33}=1 / s$, and $p_{55}=1 / s$. From Fig. 11, one can see that a $\operatorname{Tx}(3)$ of $M 2$ overlaps with a $\operatorname{Tx}(1)$, an $\operatorname{Rx}(1)$, and a $\operatorname{Tx}(1)$ of M1, in which the packet interference probability is $3 / s$, and the following $\operatorname{Rx}(1)$ overlaps with an $\operatorname{Rx}(1)$ of $M 1$, in which the packet interference probability is $1 / s$. The same situation repeats for every $\operatorname{Tx}(3)$ and $\operatorname{Rx}(1)$ of $\mathrm{M} 2$, and the average interference probability is, thus, $(3 / s+1 / s) / 2=2 / s$. We, thus, have $p_{31}=2 / s$. Using the same figure, we can also validate that $p_{13}=1 / \mathrm{s}$. Also, from Fig. 12, one can see that a $\operatorname{Tx}(5)$ of M2 overlaps with a $\operatorname{Tx}(1)$, an $\operatorname{Rx}(1), a \operatorname{Tx}(1)$, an $\operatorname{Rx}(1)$, and a $\operatorname{Tx}(1)$ of M1, in which the packet interference probability is $5 / s$, and the following $\operatorname{Rx}(1)$ overlaps with an $\operatorname{Rx}(1)$ of M1, in which the packet interference probability is $1 / s$. The same situation repeats for every $\operatorname{Tx}(5)$ and $\operatorname{Rx}(1)$ of $\mathrm{M} 2$, and the average interference probability is, thus, $(5 / s+1 / s) / 2=3 / s$. We, thus, have $p_{51}=3 / s$. Using the same figure, we can also validate that $p_{15}=1 / s$. To validate $p_{53}=2 / s$, we use

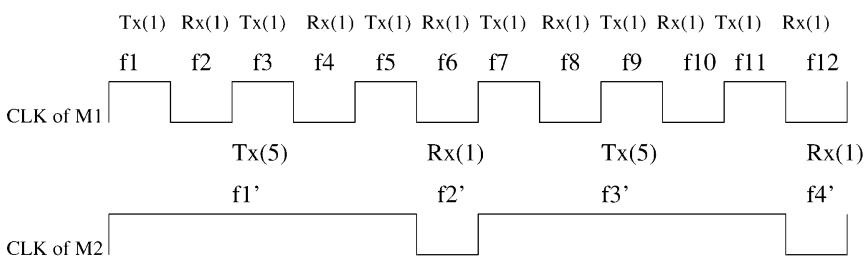

Fig. 12. Interference between two asymmetric, synchronized piconets (1- and 5-slot packets).

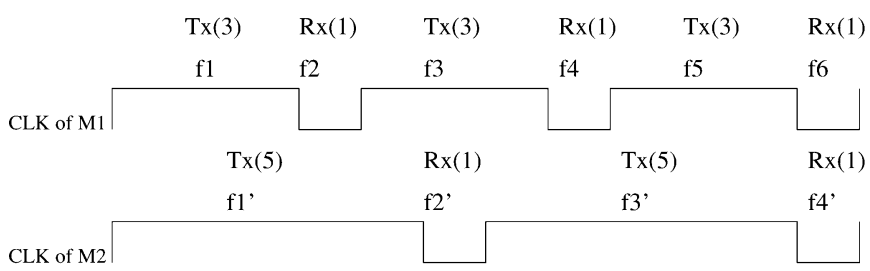

Fig. 13. Interference between two asymmetric, synchronized piconets (3- and 5-slot packets).

Fig. 13. From Fig. 13, one can see that a Tx(5) of M2 overlaps with a $\operatorname{Tx}(3)$, an $\operatorname{Rx}(1)$, and a $\operatorname{Tx}(3)$ of $M 1$, in which the interference probability is $3 / s$, and the following $\operatorname{Rx}(1)$ overlaps with a $\operatorname{Tx}(3)$ of $M 1$, in which the interference probability is $1 / s$. Then, the next $\operatorname{Tx}(5)$ overlaps with a $\operatorname{Tx}(3)$, an $\operatorname{Rx}(1)$, and a $\operatorname{Tx}(3)$ of $M 1$, in which the interference probability is $3 / s$, and then the following $\operatorname{Rx}(1)$ overlaps with the $\operatorname{Rx}(1)$ of $M 1$, in which the interference probability is $1 / s$. Then, exactly the same situation repeats for every two consecutive pairs of Tx(5) and $\operatorname{Rx}(1)$ of $\mathrm{M} 2$. Thus, the average interference probability is $(3 / s+1 / s+3 / s+1 / s) / 4=2 / s$. We, thus, have $p_{53}=2 / s$. In a similar way and using the same figure, one can also verify that $p_{35}=(1 / s+1 / s+3 / s+1 / s+1 / s+1 / s) / 6=4 / 3 s$.

2) Two Piconets Are Asynchronous: When the two piconets operate "asynchronously," we have the following lemma.

Lemma 3.4: Under asymmetric traffic, if the two Masters M1 and M2 operate asynchronously, we have $p_{11}=2 / s, p_{33}=2 / s, p_{55}=2 / s, p_{13}=3 / 2 s, p_{31}=3 / s$, $p_{15}=4 / 3 s, p_{51}=4 / s, p_{35}=5 / 3 s$, and $p_{53}=5 / 2 s$.

Proof: Again, using Fig. 5, we can easily see that $p_{11}=$ $2 / s$. To prove $p_{33}=2 / s$, we have the following four possible cases (see Fig. 14).

Case 1) $(0<$ shift $<1$-slot $)$ : A $\operatorname{Tx}(3)$ of M2 overlaps with a $\operatorname{Tx}(3)$ and an $\operatorname{Rx}(1)$ of $M 1$, in which the interference probability is $2 / s$, and then the following $\operatorname{Rx}(1)$ of M2 overlaps with an $\operatorname{Rx}(1)$ and a $\operatorname{Tx}(3)$ of $\mathrm{M} 1$, in which the interference probability is $2 / s$. Then, exactly the same situation repeats for every consecutive $\operatorname{Tx}(3)$ and $\operatorname{Rx}(1)$ of $M 2$. Thus, the interference probability is $2 / s$ for this case.

Case 2) (1-slot $<$ shift $<2$-slot): A Tx(3) of M2 overlaps with a $\operatorname{Tx}(3)$, an $\operatorname{Rx}(1)$, and a $\operatorname{Tx}(3)$ of M1, in which the interference probability is $3 / \mathrm{s}$, and then the following $\operatorname{Rx}(1)$ of $\mathrm{M} 2$ overlaps with a $\operatorname{Tx}(3)$ of M1, in which the interference probability is $1 / s$. Then, the same situation repeats for every consecutive $\operatorname{Tx}(3)$ and $\mathrm{Rx}(1)$ of M2. Thus, the average interference probability for this case is $(3 / s+1 / s) / 2=2 / s$. 


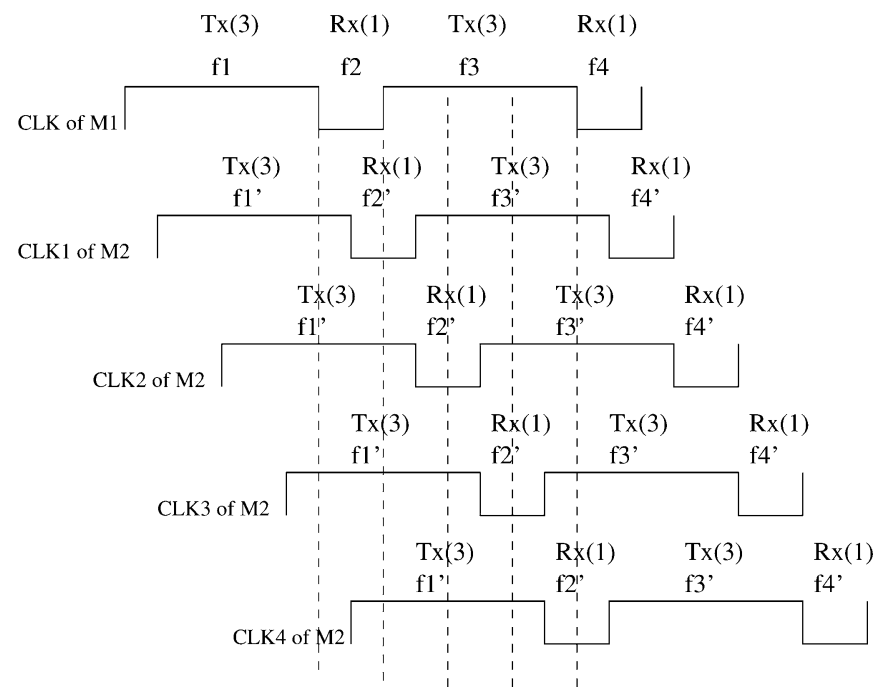

Fig. 14. Interference between two asymmetric, asynchronous piconets (3-slot packets).

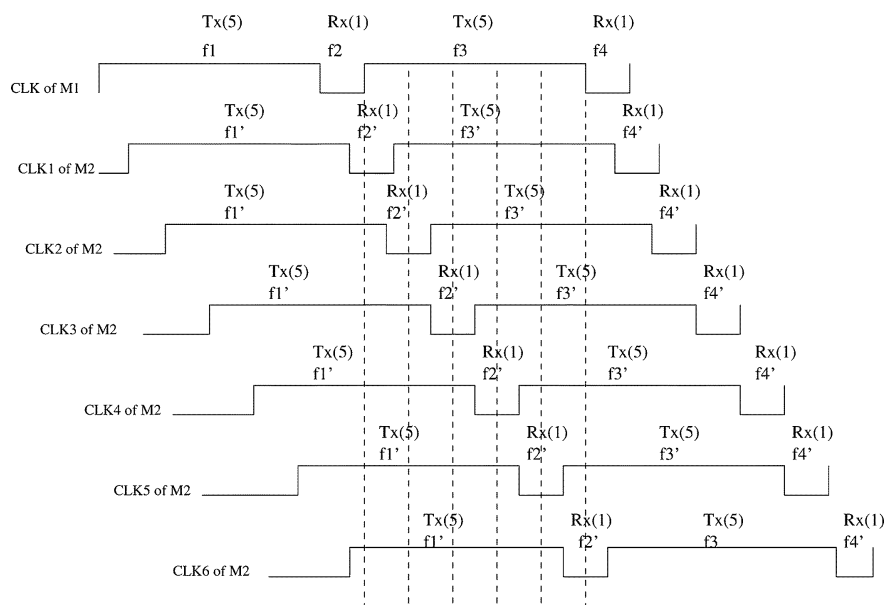

Fig. 15. Interference between two asymmetric, asynchronous piconets (5-slot packets).

Case 3) (2-slot $<$ shift $<3$-slot): Similar to Case 2) and the probability is $2 / s$.

Case 4) (3-slot $<$ shift $<4$-slot): A Tx(3) of M2 overlaps with an $\operatorname{Rx}(1)$ and a $\operatorname{Tx}(3)$ of M1, in which the interference probability is $2 / s$, and then the following $\operatorname{Rx}(1)$ of M2 overlaps with a $\operatorname{Tx}(3)$ and an $\operatorname{Rx}(1)$ of M1, in which the interference probability is $2 / s$. Then, exactly the same situation repeats for every consecutive $\operatorname{Tx}(3)$ and $\operatorname{Rx}(1)$ of M2. Thus, the interference probability is $2 / s$ for this case.

Therefore, $p_{33}=1 / 4 \times 2 / s+1 / 4 \times 2 / s+1 / 4 \times 2 / s+$ $1 / 4 \times 2 / s=2 / s$.

We now use Fig. 15 to prove that $p_{55}=2 / s$. There are six cases.

Case 1) $(0<$ shift $<1$-slot $)$ : A Tx(5) of M2 overlaps with a $\operatorname{Tx}(5)$ and an $\operatorname{Rx}(1)$ of M1, in which the interference probability is $2 / s$, and the following $\operatorname{Rx}(1)$ of M2 overlaps with an $\operatorname{Rx}(1)$ and a $\operatorname{Tx}(5)$ of M1, in which the interference probability is $2 / s$. Then, the same situation repeats for the subsequent $\mathrm{Tx}(5) \mathrm{s}$

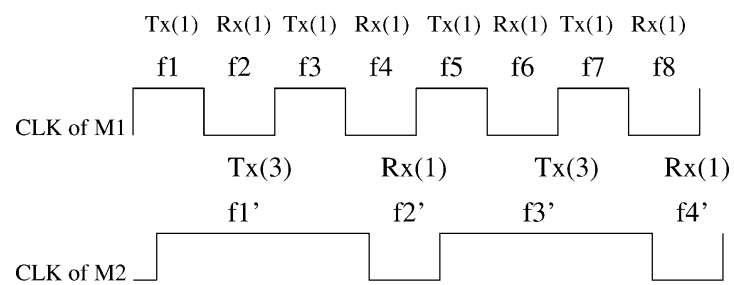

Fig. 16. Interference between two asymmetric, asynchronous piconets (1- and 3-slot packets).

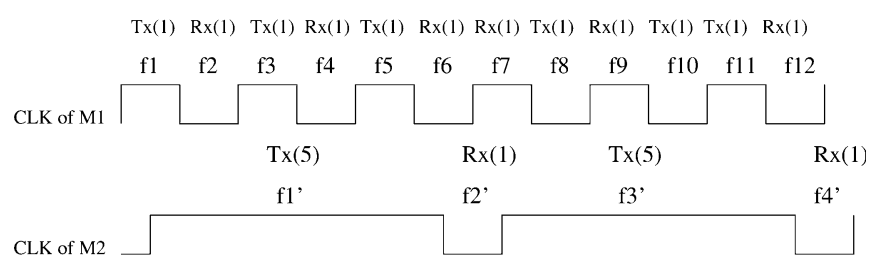

Fig. 17. Interference between two asymmetric, asynchronous piconets (1- and 5-slot packets).

and $\operatorname{Rx}(1) s$ of M2. Thus, the interference probability is $2 / s$ for this case.

Case 2) (1-slot $<$ shift $<2$-slot): A Tx(5) of M2 overlaps with a $\operatorname{Tx}(5)$, an $\operatorname{Rx}(1)$, and a $\operatorname{Tx}(5)$ of M1, in which the interference probability is $3 / s$. Then, the following $\operatorname{Rx}(1)$ of $\mathrm{M} 2$ overlaps with a $\mathrm{Tx}(5)$ of M1, in which the interference probability is $1 / s$. Then, the same situation repeats for the subsequent $\mathrm{Tx}(5) \mathrm{s}$ and $\mathrm{Rx}(1) \mathrm{s}$ of $\mathrm{M} 2$. Thus, the average interference probability for this case is $(3 / s+1 / s) / 2=2 s$.

Case 3) (2-slot $<$ shift $<3$-slot): Similar to Case 2) and the probability is $2 / s$.

Case 4) (3-slot $<$ shift $<4$-slot): Similar to Case 2) and the probability is $2 / s$.

Case 5) (4-slot $<$ shift $<5$-slot)): Similar to Case 2) and the probability is $2 / s$.

Case 6) (5-slot $<$ shift $<6$-slot): A Tx(5) of M2 overlaps with an $\operatorname{Rx}(1)$ and a $\operatorname{Tx}(5)$ of M1, in which the interference probability is $2 / s$, and the following $\operatorname{Rx}(1)$ of M2 overlaps with a $\operatorname{Tx}(5)$ and an $\operatorname{Rx}(1)$ of $\mathrm{M} 1$, in which the interference probability is $2 / s$. Then, the same situation repeats for the subsequent $\mathrm{Tx}(5) \mathrm{s}$ and $\mathrm{Rx}(1) \mathrm{s}$ of M2. Thus, the interference probability is $2 / s$ for this case.

Therefore, $p_{55}=1 / 6 \times 2 / s+1 / 6 \times 2 / s+1 / 6 \times 2 / s+$ $1 / 6 \times 2 / s+1 / 6 \times 2 / s+1 / 6 \times 2 / s=2 / s$.

There is only one possible shift, i.e., $0<$ shift $<1$-slot in proving $p_{31}=3 / s$ (see Fig. 16). A Tx(3) of M2 overlaps with four 1-slot packets of M1, and the following $\operatorname{Rx}(1)$ overlaps with two 1-slot packets of M1. The interference probability is, thus, $(4 / s+2 / s) / 2=3 / s$.

Using the same figure, one can also show that $p_{13}=(2 / s+$ $1 / s+1 / s+2 / s) / 4=3 / 2 s$.

Also, there is only one possible shift, i.e., $0<$ shift $<1$-slot in proving $p_{51}=4 / s$ (see Fig. 17). A Tx(5) of M2 overlaps with six 1-slot packets of M1, and the following Rx(1) of M2 overlaps with two 1-slot packets of M1. The interference probability of $p_{51}$ is, thus, $(6 / s+2 / s) / 2=4 / s$. 


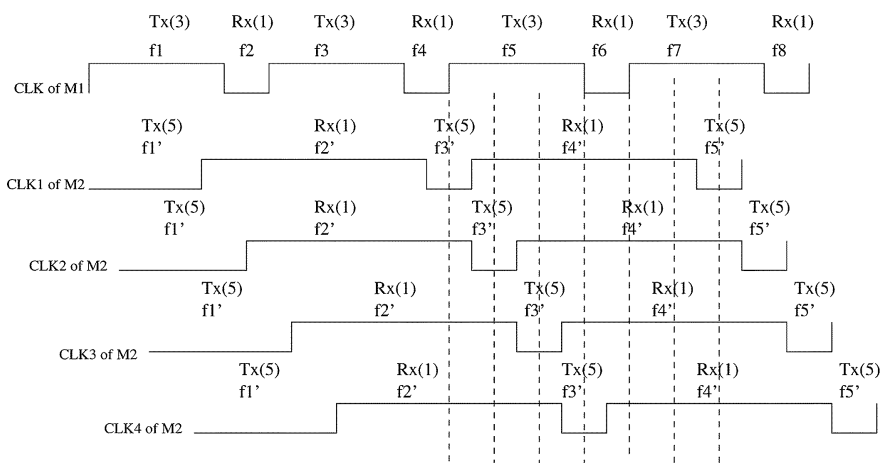

Fig. 18. Interference between two asymmetric, asynchronous piconets (3- and 5-slot packets).

Using the same figure, one can also show that $p_{15}=(2 / s+$ $1 / s+1 / s+1 / s+1 / s+2 / s) / 6=4 / 3 s$.

We use Fig. 18 to prove that $p_{53}=5 / 2 s$. There are four cases.

Case 1) $(0<$ shift $<1$-slot $)$ : In this case, a Tx(5) of M2 overlaps with a $\operatorname{Tx}(3)$, an $\operatorname{Rx}(1)$, and a $\operatorname{Tx}(3)$ of M1, in which the interference probability is $3 / s$, and the following $\operatorname{Rx}(1)$ of M2 overlaps with a $\operatorname{Tx}(3)$ of $\mathrm{M} 1$, in which the interference probability is $1 / s$. Then, the next $\operatorname{Tx}(5)$ of M2 overlaps with a $\operatorname{Tx}(3)$, an $\operatorname{Rx}(1)$, a $\operatorname{Tx}(3)$, and an $\operatorname{Rx}(1)$ of $\mathrm{M} 1$, in which the interference probability is $4 / s$. Then, the following $\operatorname{Rx}(1)$ of M2 overlaps with an $\operatorname{Rx}(1)$ and a $\operatorname{Tx}(3)$ of M1, in which the interference probability is $2 / s$. Then, the same situation will be repeated for the subsequent $\mathrm{Tx}(5) \mathrm{s}$ and $\mathrm{Rx}(1) \mathrm{s}$ of M2. Thus, the average interference probability for this case is $(3 / s+1 / s+4 / s+2 / s) / 4=5 / 2 s$.

Case 2) (1-slot $<$ shift $<2$-slot): In this case, a Tx(5) of M2 overlaps with a $\operatorname{Tx}(3)$, an $\operatorname{Rx}(1)$, and a $\operatorname{Tx}(3)$ of M1, in which the interference probability is $3 / s$, and the following $\operatorname{Rx}(1)$ of M2 overlaps with a Tx(3) and an $\mathrm{Rx}(1)$ of M1, in which the interference probability is $2 / s$. Then, the next $\operatorname{Tx}(5)$ of M2 overlaps with an $\operatorname{Rx}(1)$, a $\operatorname{Tx}(3)$, an $\operatorname{Rx}(1)$, and a $\operatorname{Tx}(3)$ of $\mathrm{M} 1$, in which the interference probability is $4 / s$. Then, the following $\operatorname{Rx}(1)$ of M2 overlaps with a Tx(3) of M1, in which the interference probability is $1 / s$. Then, the above same situation repeats. Thus, the average interference probability for this case is $(3 / s+2 / s+$ $4 / s+1 / s) / 4=5 / 2 s$.

Case 3) (2-slot $<$ shift $<3$-slot): In this case, a Tx(5) of M2 overlaps with a $\operatorname{Tx}(3)$, an $\operatorname{Rx}(1)$, a $\operatorname{Tx}(3)$, and an $\operatorname{Rx}(1)$ of $\mathrm{M} 1$, in which the interference probability is $4 / s$, and the following $\operatorname{Rx}(1)$ of $\mathrm{M} 2$ overlaps with an $\operatorname{Rx}(1)$ and a Tx(3) of M1, in which the interference probability is $2 / s$. Then, the next $\operatorname{Tx}(5)$ of M2 overlaps with a $\operatorname{Tx}(3)$, an $\operatorname{Rx}(1)$, and a $\operatorname{Tx}(3)$ of $\mathrm{M} 1$, in which the interference probability is $3 / s$. Then, the following $\operatorname{Rx}(1)$ of M2 overlaps with a $\operatorname{Tx}(3)$ of M1, in which the interference probability is $1 / s$. Then, exactly the same situation repeats. Thus, the average interference probability for this case is $(4 / s+2 / s+3 / s+1 / s) / 4=5 / 2 s$.
Case 4) $\quad$ (3-slot < shift < 4-slot): In this case, a $\operatorname{Tx}(5)$ of M2 overlaps with an $\operatorname{Rx}(1)$, a $\operatorname{Tx}(3)$, an $\operatorname{Rx}(1)$, and $\operatorname{Tx}(3)$ of M1, in which the interference probability is $4 / s$, and the following $\operatorname{Rx}(1)$ of M2 overlaps with a $\operatorname{Tx}(3)$ of $\mathrm{M} 1$, in which the interference probability is $1 / s$. Then, the next $\mathrm{Tx}(5)$ of M2 overlaps with a $\operatorname{Tx}(3)$, an $\operatorname{Rx}(1)$, and a $\operatorname{Tx}(3)$ of $M 1$, in which the interference probability is $3 / s$. Then, the following $\operatorname{Rx}(1)$ of $\mathrm{M} 2$ overlaps with a $\operatorname{Tx}(3)$ and an $\operatorname{Rx}(1)$ of M1, in which the interference probability is $2 / s$. Then, exactly the same situation repeats. Thus, the average interference probability for this case is $(4 / s+1 / s+3 / s+2 / s) / 4=5 / 2 s$.

We, thus, conclude that $p_{53}=1 / 4 \times 5 / 2 s+1 / 4 \times 5 / 2 s+$ $1 / 4 \times 5 / 2 s+1 / 4 \times 5 / 2 s=5 / 2 s$. In a similar way and using the same figure, one can also validate that $p_{35}=5 / 3 \mathrm{~s}$.

\section{The Model}

In a cluster of $N$ piconets of a homogeneous system, we have three types of nodes, namely 1 -slot type, 3-slot-type, and 5-slot type. Let $N_{\alpha}$ be the number of piconets which transmit 1-slot packets, $N_{\beta}$ be the number of piconets which transmit 3-slot packets, and $N_{\gamma}$ be the number of piconets which transmit 5-slot packets. We have $N=N_{\alpha}+N_{\beta}+N_{\gamma}$. We now construct a probabilistic graph $G$ of $N$ nodes in the same way as that stated in the previous section. Also, let $X_{N}$ be the random variable of the number of nodes having no any incident edge. We wanted to develop the model for $E\left(X_{N}\right)$.

Lemma 3.5: Given a probabilistic graph $G$ as defined above, let $p_{\alpha}$ be the probability that a node of the 1-slot type has no incident edge, $p_{\beta}$ be the probability that a node of the 3-slot type has no incident edge, and $p_{\gamma}$ be the probability that a node of the 5-slot type has no incident edge. Also, let $p_{i j}$ be the packet interference rates defined in Lemma 3.1, or Lemma 3.2, or Lemma 3.3, or Lemma 3.4. Then, we have $p_{\alpha}=\left(1-p_{11}\right)^{N_{\alpha}-1} \cdot(1-$ $\left.p_{13}\right)^{N_{\beta}} \cdot\left(1-p_{15}\right)^{N_{\gamma}}, p_{\beta}=\left(1-p_{31}\right)^{N_{\alpha}} \cdot\left(1-p_{33}\right)^{N_{\beta}-1} \cdot(1-$ $\left.p_{35}\right)^{N_{\gamma}}$, and $p_{\gamma}=\left(1-p_{51}\right)^{N_{\alpha}} \cdot\left(1-p_{53}\right)^{N_{\beta}} \cdot\left(1-p_{55}\right)^{N_{\gamma}-1}$

Proof: A node of the 1-slot type has $N_{\alpha}-1$ possible links to the nodes of the same type, and has $N_{\beta}$ possible links to the nodes of the 3-slot type, and has $N_{\gamma}$ possible links to the nodes of the 5-slot type. We, thus, have $p_{\alpha}=\left(1-p_{11}\right)^{N_{\alpha}-1} \cdot(1-$ $\left.p_{13}\right)^{N_{\beta}} \cdot\left(1-p_{15}\right)^{N_{\gamma}}$. Also, a node of the 3-slot type has $N_{\alpha}$ possible links to the nodes of the 1-slot type, and has $N_{\beta}-1$ possible links to the nodes of the same type, and has $N_{\gamma}$ possible links to the nodes of the 5-slot type. We, thus, have $p_{\beta}=(1-$ $\left.p_{31}\right)^{N_{\alpha}} \cdot\left(1-p_{33}\right)^{N_{\beta}-1} \cdot\left(1-p_{35}\right)^{N_{\gamma}}$. Finally, since a node of the 5-slot type has $N_{\alpha}$ possible links to the nodes of the 1-slot type, and has $N_{\beta}$ possible links to the nodes of the 3-slot type, and has $N_{\gamma}-1$ possible links to the nodes of the same type, we have $p_{\gamma}=\left(1-p_{51}\right)^{N_{\alpha}} \cdot\left(1-p_{53}\right)^{N_{\beta}} \cdot\left(1-p_{55}\right)^{N_{\gamma}-1}$.

We are now ready to validate the following theorem.

Theorem 3.1: Let $G$ be a probabilistic graph with $N_{\alpha}$ nodes of the 1-slot type, $N_{\beta}$ nodes of the 3-slot type, and $N_{\gamma}$ nodes of the 5-slot type, where the weights of edges (interference rates between nodes) are specified as in Lemma 3.1, or Lemma 3.2, or Lemma 3.3, or Lemma 3.4. Also, let $E\left(X_{N}\right)$ be the expected 
number of nodes of all types having no incident edge. We can represent $E\left(X_{N}\right)$ as follows:

$$
\begin{aligned}
E\left(X_{N}\right)=\sum_{k=1}^{N} k \cdot\left(\sum_{r_{\alpha}=}\right. & \max \left(0, k-\left(N_{\beta}+N_{\gamma}\right)\right) \\
& \left.\cdot\left(\sum_{r_{\beta}=\max \left(0,\left(k-r_{\alpha}\right)-N_{\gamma}\right)}^{\min \left(N_{\alpha}, k\right)} X_{\beta} \cdot X_{\gamma}\right)\right)
\end{aligned}
$$

where

$$
\begin{aligned}
X_{\alpha} & =\left(\begin{array}{c}
N_{\alpha} \\
r_{\alpha}
\end{array}\right) p_{\alpha}^{r_{\alpha}}\left(1-p_{\alpha}\right)^{N_{\alpha}-r_{\alpha}} \\
X_{\beta} & =\left(\begin{array}{c}
N_{\beta} \\
r_{\beta}
\end{array}\right) p_{\beta}^{r_{\beta}}\left(1-p_{\beta}\right)^{N_{\beta}-r_{\beta}} \\
X_{\gamma} & =\left(\begin{array}{c}
N_{\gamma} \\
\left(k-r_{\alpha}\right)-r_{\beta}
\end{array}\right) p_{\gamma}^{\left(k-r_{\alpha}\right)-r_{\beta}}\left(1-p_{\gamma}\right)^{N_{\gamma}-\left(\left(k-r_{\alpha}\right)-r_{\beta}\right)}
\end{aligned}
$$

$k=r_{\alpha}+r_{\beta}+r_{\gamma}, r_{\alpha}$ is the number of nodes of the 1-slot type having no incident edge, $r_{\beta}$ is the number of nodes of the 3 -slot type having no incident edge, and $r_{\gamma}$ is the number of nodes of the 5-slot type having no incident edge.

Proof: Let random variable $X_{N}$ be the number of nodes of any type with no incident edge in the graph. Also, WLOG, let random variable $X_{N_{\alpha}}$ be the number of nodes of the 1-slot type with no incident edge, random variable $X_{N_{\beta}}$ be the number of nodes of the 3-slot type with no incident edge, and random variable $X_{N_{\gamma}}$ be the number of nodes of the 5-slot type with no incident edge. Since $X_{N_{\alpha}}, X_{N_{\beta}}$, and $X_{N_{\gamma}}$ are independent random variables, we have $X_{N}=X_{N_{\alpha}}+X_{N_{\beta}}+X_{N_{\gamma}}$. Thus, $X_{N}=k$ if and only if $X_{N_{\alpha}}=r_{\alpha}$ and $X_{N_{\beta}}+X_{N_{\gamma}}=k-r_{\alpha}$, for any integer $r_{\alpha}$ satisfying $0 \leq r_{\alpha} \leq N_{\alpha}$ and $0 \leq k-$ $r_{\alpha} \leq N_{\beta}+N_{\gamma}$. Also, $X_{N_{\beta}}+X_{N_{\gamma}}=k-r_{\alpha}$ if and only if $X_{N_{\beta}}=r_{\beta}$ and $X_{N_{\gamma}}=\left(k-r_{\alpha}\right)-r_{\beta}$, for any integer $r_{\beta}$ satisfying $0 \leq r_{\beta} \leq N_{\beta}$ and $0 \leq\left(k-r_{\alpha}\right)-r_{\beta} \leq N_{\gamma}$. The above restrictions on $r_{\alpha}$ are equivalent to $0 \leq r_{\alpha} \leq N_{\alpha}$ and $k-\left(N_{\beta}+N_{\gamma}\right) \leq r_{\alpha} \leq k$. Combining these restrictions, we can write $\max \left(0, k-\left(N_{\beta}+N_{\gamma}\right)\right) \leq r_{\alpha} \leq \min \left(N_{\alpha}, k\right)$. Also, the restrictions on $r_{\beta}$ are equivalent to $0 \leq r_{\beta} \leq N_{\beta}$ and $\left(k-r_{\alpha}\right)-N_{\gamma} \leq r_{\beta} \leq k-r_{\alpha}$. Combining these restrictions, we can write $\max \left(0,\left(k-r_{\alpha}\right)-N_{\gamma}\right) \leq r_{\beta} \leq \min \left(N_{\beta}, k-r_{\alpha}\right)$. With Lemma 3.5, we, thus, have

$$
\begin{aligned}
P\left(X_{N}=k\right)= & \sum_{r_{\alpha}=\max \left(0, k-\left(N_{\beta}+N_{\gamma}\right)\right)}^{\min \left(N_{\alpha}, k\right)} X_{\alpha} \\
& \cdot\left(\sum_{r_{\beta}=\max \left(0,\left(k-r_{\alpha}\right)-N_{\gamma}\right)}^{\min \left(N_{\beta}, k-r_{\alpha}\right)} X_{\beta} \cdot X_{\gamma}\right)
\end{aligned}
$$

where

$$
\begin{aligned}
X_{\alpha} & =\left(\begin{array}{c}
N_{\alpha} \\
r_{\alpha}
\end{array}\right) p_{\alpha}^{r_{\alpha}}\left(1-p_{\alpha}\right)^{N_{\alpha}-r_{\alpha}} \\
X_{\beta} & =\left(\begin{array}{c}
N_{\beta} \\
r_{\beta}
\end{array}\right) p_{\beta}^{r_{\beta}}\left(1-p_{\beta}\right)^{N_{\beta}-r_{\beta}}
\end{aligned}
$$

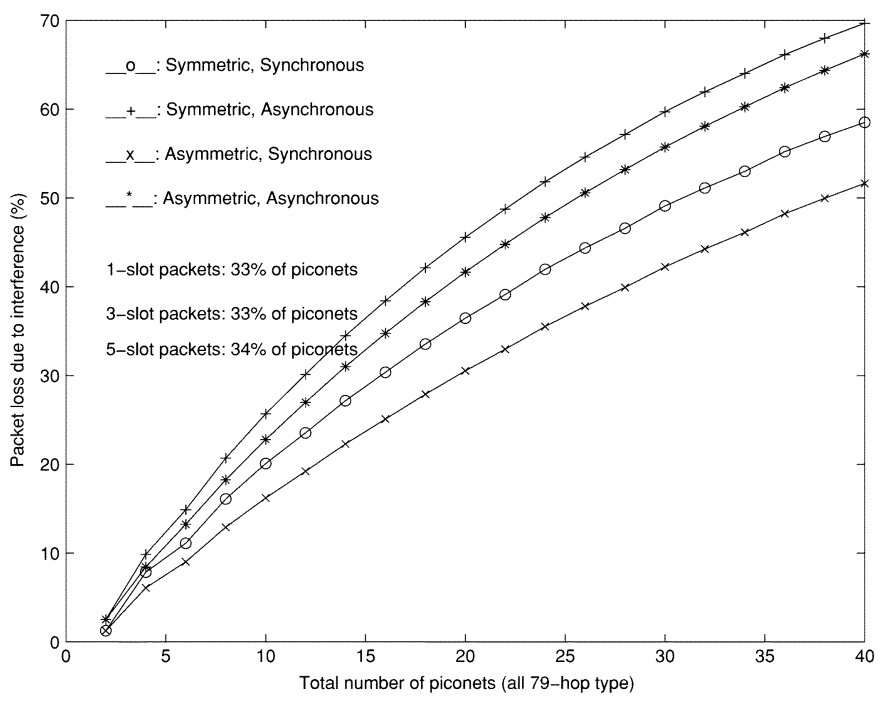

Fig. 19. Packet loss in a cluster of 33\% 1-slot type piconets, 33\% 3-slot type piconets, and 34\% 5-slot type piconets.

and

$X_{\gamma}=\left(\begin{array}{c}N_{\gamma} \\ \left(k-r_{\alpha}\right)-r_{\beta}\end{array}\right) p_{\gamma}^{\left(k-r_{\alpha}\right)-r_{\beta}}\left(1-p_{\gamma}\right)^{N_{\gamma}-\left(\left(k-r_{\alpha}\right)-r_{\beta}\right)}$.

Therefore, $E\left(X_{N}\right)=\sum_{k=1}^{N} k \cdot P\left(X_{N}=k\right)$.

The developed model can be used for symmetric synchronous system, or symmetric asynchronous system, or asymmetric synchronous system, or asymmetric asynchronous system, by simply plugging the $p_{i j}$ 's of Lemma 3.1, or Lemma 3.2, or Lemma 3.3, or Lemma 3.4, respectively, into the model.

Fig. 19 is drawn using the model of Theorem 3.1 for a cluster of 79-hop type with $1 / 3$ of 1 -slot packets, $1 / 3$ of 3 -slot packets, and $1 / 3$ of 5 -slot packets. From the plot, we see the following.

- Under the same type of traffic, i.e., either symmetric or asymmetric, the packet loss of a synchronous system is always lower than that of an asynchronous one, which can be easily predicted.

- Whether the piconets operate synchronously or asynchronously, the packet loss under asymmetric traffic is likely lower than that under symmetric traffic.

However, we are also interested in the aggregated throughput of a cluster of piconets. Unfortunately, Theorem 3.1 does not provide enough information for calculating the aggregated throughput. Since different types of packets carry different number of bits, to calculate the throughput, we need first to know $E\left(X_{N_{\alpha}}\right)$ (the expected number of uninterfered 1-slot type nodes), $E\left(X_{N_{\beta}}\right)$ (the expected number of uninterfered 3-slot type nodes), and $E\left(X_{N_{\gamma}}\right)$ (the expected number of uninterfered 5-slot type nodes). Fortunately, using the fact that $X_{N_{\alpha}}, X_{N_{\beta}}$, and $X_{N_{\gamma}}$ are independent random variables, the following theorem can be proved.

Theorem 3.2: Let $G$ be a probabilistic graph with $N_{\alpha}$ nodes of the 1-slot type, $N_{\beta}$ nodes of the 3-slot type, and $N_{\gamma}$ nodes of the 5-slot type, where the probabilities of edges between nodes are specified as in Lemma 3.1, or Lemma 3.2, or Lemma 3.3, or Lemma 3.4. Also, let $E\left(X_{N}\right)$ be the expected number of 
nodes of all types having no incident edge, $E\left(X_{N_{\alpha}}\right)$ be the expected number of nodes of 1-slot type having no incident edge, $E\left(X_{N_{\beta}}\right)$ be the expected number of nodes of 3-slot type having no incident edge, and $E\left(X_{N_{\gamma}}\right)$ be the expected number of nodes of 5-slot type having no incident edge. We have

$$
\begin{aligned}
& E\left(X_{N_{\alpha}}\right)=\sum_{r_{\alpha}=1}^{N_{\alpha}} r_{\alpha} \cdot\left(\begin{array}{c}
N_{\alpha} \\
r_{\alpha}
\end{array}\right) p_{\alpha}^{r_{\alpha}}\left(1-p_{\alpha}\right)^{N_{\alpha}-r_{\alpha}} \\
& E\left(X_{N_{\beta}}\right)=\sum_{r_{\beta}=1}^{N_{\beta}} r_{\beta} \cdot\left(\begin{array}{c}
N_{\beta} \\
r_{\beta}
\end{array}\right) p_{\beta}^{r_{\beta}}\left(1-p_{\beta}\right)^{N_{\beta}-r_{\beta}} \\
& E\left(X_{N_{\gamma}}\right)=\sum_{r_{\gamma}=1}^{N_{\gamma}} r_{\gamma} \cdot\left(\begin{array}{c}
N_{\gamma} \\
r_{\gamma}
\end{array}\right) p_{\gamma}^{r_{\gamma}}\left(1-p_{\gamma}\right)^{N_{\gamma}-r_{\gamma}}
\end{aligned}
$$

and

$$
E\left(X_{N}\right)=E\left(X_{N_{\alpha}}\right)+E\left(X_{N_{\beta}}\right)+E\left(X_{N_{\gamma}}\right)
$$

where $p_{\alpha}, p_{\beta}$, and $p_{\gamma}$ are defined in Lemma 3.5.

When calculating the aggregated throughput for a a cluster of piconets using multiple-slot packets, for more accurate throughput, we had better follow the following calculations:

$$
\begin{aligned}
& \text { 1-slot packets: } \\
& \text { total duration }=625 \mu \mathrm{s} \\
& \text { payload }=240 \text { bits ( } 30 \text { bytes }) \\
& \text { 3-slot packets: } \\
& \text { total duration }=3 * 625=1875 \mu \mathrm{s} \\
& \text { payload }=1464 \text { bits ( } 183 \text { bytes }) \\
& \text { 5-slot packets: } \\
& \text { total duration }=5 * 625=3125 \mu \mathrm{s} \\
& \text { payload }=2712 \text { bits }(339 \text { bytes }) .
\end{aligned}
$$

Then, the aggregated throughput of the system using symmetric traffic can be given by the following expression:

$$
\begin{aligned}
& \text { Aggregated throughput } \\
& \begin{aligned}
= & E\left(X_{N_{\alpha}}\right) \times 10^{6} \times(240 / 625) \\
& +E\left(X_{N_{\beta}}\right) \times 10^{6} \times(1464 / 1875) \\
& +E\left(X_{N_{\gamma}}\right) \times 10^{6} \times(2712 / 3125) \text { bits/s. }
\end{aligned}
\end{aligned}
$$

Also, the aggregated throughput of the system using asymmetric traffic can be given by the following expression:

$$
\begin{aligned}
& \text { Aggregated throughput } \\
& \begin{aligned}
= & E\left(X_{N_{\alpha}}\right) \times 10^{6} \times(240 / 625) \\
& +E\left(X_{N_{\beta}}\right) \times 10^{6} \times((1464 / 1875)+(240 / 625)) / 2 \\
& +E\left(X_{N_{\gamma}}\right) \times 10^{6} \times((2712 / 3125) \\
& +(240 / 625)) / 2 \text { bits/s. }
\end{aligned}
\end{aligned}
$$

The aggregated throughputs of various combinations of different types of Masters under different traffic conditions are shown in Figs. 20-24. We interpret these figures in what follows.

- In general, if the piconets operate synchronously, it produces higher aggregated throughput under either sym-

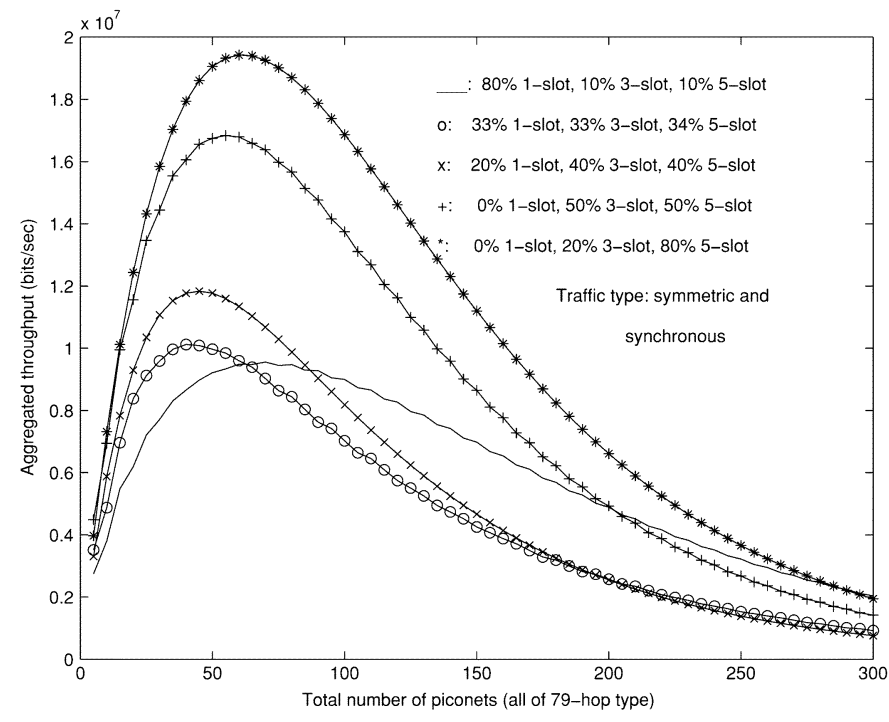

Fig. 20. Aggregated throughputs of various symmetric traffic when all piconets are of 79-hop type and are all synchronized.

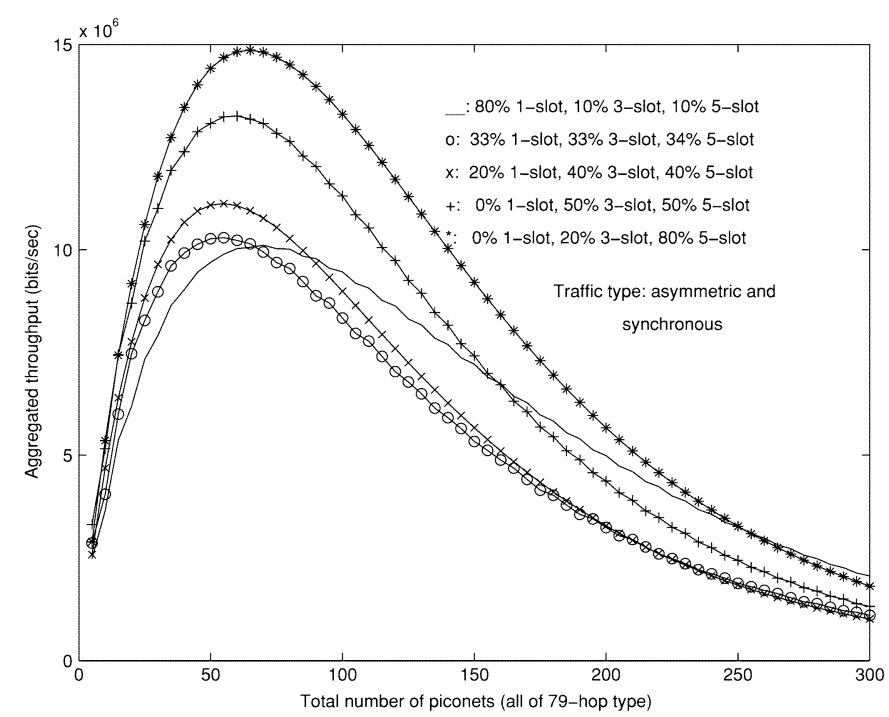

Fig. 21. Aggregated throughputs of various asymmetric traffic when all piconets are of 79-hop type and are all synchronized.

metric or asymmetric traffic, compared with the unsynchronized system. It also allows us to accommodate more piconets, say around 60 , in an area to reach the maximum aggregated throughput, compared with the unsynchronized one, which can accommodate only around 40 piconets to reach its maximum aggregated throughput.

- Whether the piconets operate synchronously or asynchronously, in general, the system with symmetric traffic gain higher aggregated throughput than that gained by the one with asymmetric traffic. This is not hard to predict since twice of the one-way symmetric data rate is more than the sum of the upstream and downstream data rate for asymmetric traffic in a channel.

- If we use only 23-hop type Bluetooth devices to form a cluster of piconets, due to the much higher packet interference rate, the maximum aggregated throughput we can 


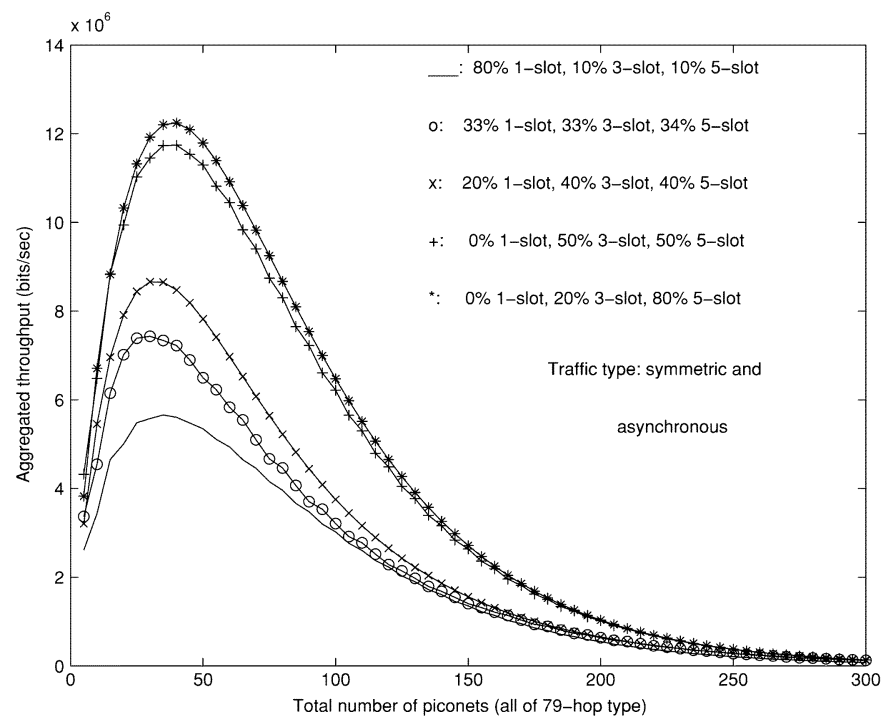

Fig. 22. Aggregated throughputs of various symmetric traffic when all piconets are of 79-hop type and operate asynchronously.

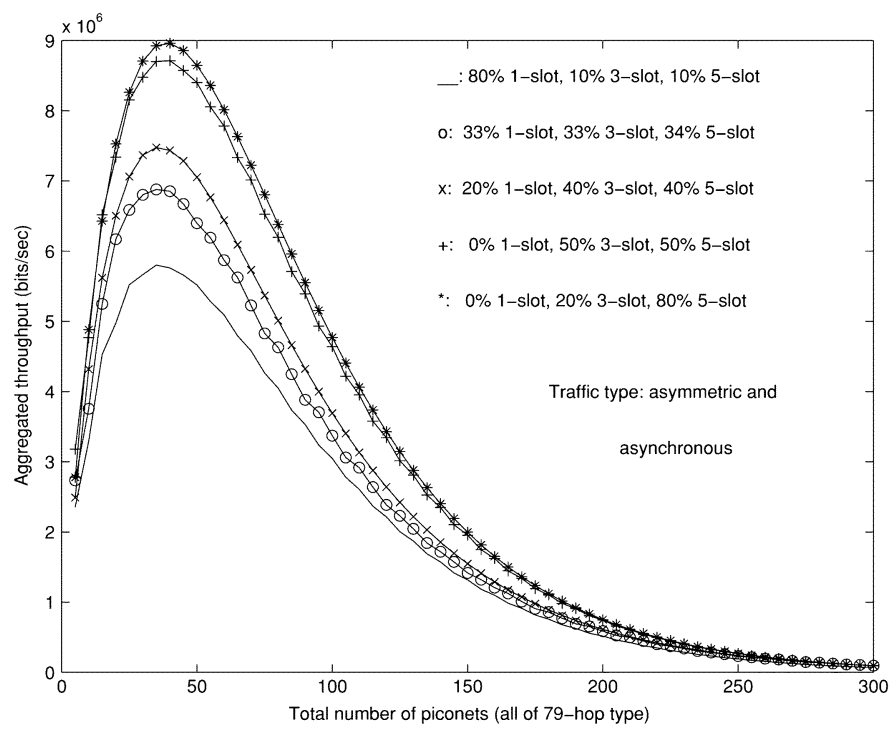

Fig. 23. Aggregated throughputs of various asymmetric traffic when all piconets are of 79-hop type and operate asynchronously.

gain is much lower than what can be gained by the cluster of piconets formed from only 79-hop type Bluetooth devices. Besides, it can accommodate only around 15 piconets to reach the maximum aggregated throughput. This means that if we are given more than $(1+7) \times 15=120$ 23-hop type Bluetooth devices in an area to form more than 15 piconets, the throughput we can gain will be extremely low.

- Consider the fact that, in reality, a cluster of piconets is likely to operate in asymmetric mode and in asynchronous condition of Master clocks, we use Fig. 23 to conclude the discussion on the model of homogeneous system. When forming a cluster of piconets in an area using a given number of 79-hop Bluetooth devices, if the given number of devices is less than $(1+7) \times 40=320$, try to form the cluster in such a way that the number of piconets is close to 40 . In case the number of Bluetooth devices in the area

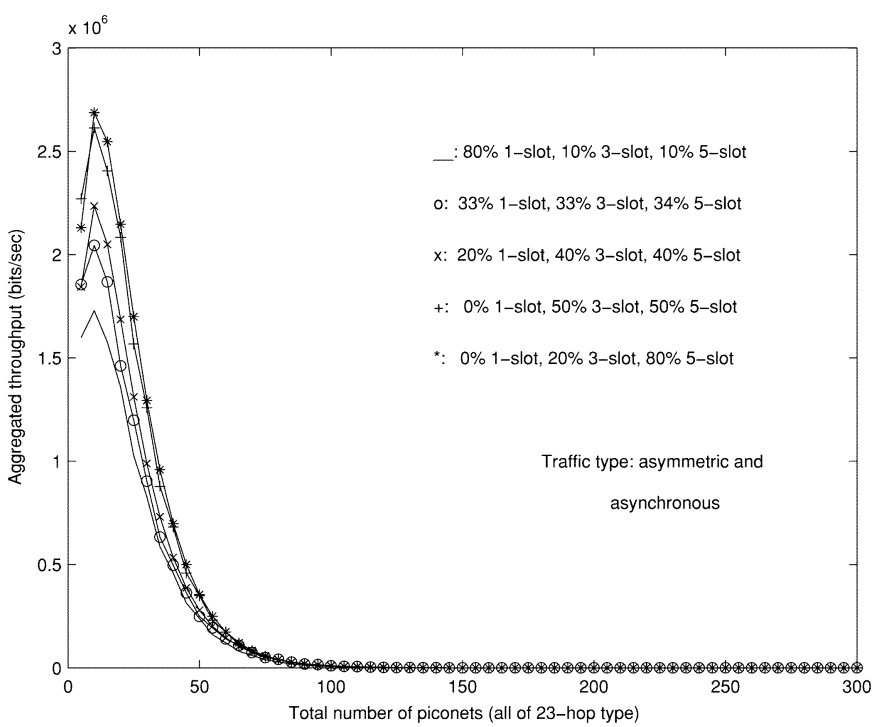

Fig. 24. Aggregated throughputs of various asymmetric traffic when all piconets are of 23-hop type and operate asynchronously.

is more than 320 , try to form the cluster of piconets with as smaller number of piconets as possible. In general, it is not a good idea to accommodate more than 50 piconets in a cluster.

\section{INTERFERENCE MODEL OF HETEROGENEOUS SYSTEM}

In a heterogeneous system, in addition to those $p_{i j}$ s calculated in the previous section, we also need to calculate the $p_{i j}^{\prime} \mathrm{s}$, the packet interference probability between a Master of 79-hop type and a Master of 23-hop type.

Lemma 4.1: If Master M1 is of 79-hop type and Master M2 is of 23-hop type, and if both M1 and M2 are synchronized and are with symmetric traffic, we have $p_{11}^{\prime}=1 / 79, p_{33}^{\prime}=1 / 79, p_{55}^{\prime}=1 / 79, p_{13}^{\prime}=1 / 79$, $p_{31}^{\prime}=3 / 79, p_{15}^{\prime}=1 / 79, p_{51}^{\prime}=5 / 79, p_{35}^{\prime}=7 /(5 \times 79)$, and $p_{53}^{\prime}=7 /(3 \times 79)$.

Proof: Tossing a die of $s 1$ sides, labeled from 1 to $s 1$, and a die of $s 2$ sides, labeled from 1 to $s 2$, and WLOG, assuming that $s 1 \geq s 2$, the probability that we obtain the same numbers from both dice is $1 / s 1$. Given this fact, the rest of the proof is similar to that of Lemma 3.1.

Lemma 4.2: If Master M1 is of 79-hop type and Master M2 is of 23-hop type, and if both M1 and M2 operate asynchronously and are with symmetric traffic, we have $p_{11}^{\prime}=2 / 79, p_{33}^{\prime}=$ $2 / 79, p_{55}^{\prime}=2 / 79, p_{13}^{\prime}=4 /(3 \times 79), p_{31}^{\prime}=4 / 79, p_{15}^{\prime}=$ $6 /(5 \times 79), p_{51}^{\prime}=6 / 79, p_{35}^{\prime}=8 /(5 \times 79)$, and $p_{53}^{\prime}=8 /(3 \times$ 79).

Proof: Using the fact given in Lemma 4.1, the rest of the proof is similar to that of Lemma 3.2.

Lemma 4.3: If Master M1 is of 79-hop type and Master M2 is of 23-hop type, and if both M1 and M2 are synchronized and are with asymmetric traffic, we have $p_{11}^{\prime}=1 / 79, p_{33}^{\prime}=1 / 79, p_{55}^{\prime}=1 / 79, p_{13}^{\prime}=1 / 79$, $p_{31}^{\prime}=2 / 79, p_{15}^{\prime}=1 / 79, p_{51}^{\prime}=3 / 79, p_{35}^{\prime}=4 /(3 \times 79)$, and $p_{53}^{\prime}=2 / 79$.

Proof: Using the fact given in Lemma 4.1, the rest of the proof is similar to that of Lemma 3.3. 
Lemma 4.4: If Master M1 is of 79-hop type and M2 is of 23-hop type, and if both M1 and M2 operate asynchronously and are with asymmetric traffic, we have $p_{11}^{\prime}=2 / 79, p_{33}^{\prime}=$ $2 / 79, p_{55}^{\prime}=2 / 79, p_{13}^{\prime}=3 /(2 \times 79), p_{31}^{\prime}=3 / 79, p_{15}^{\prime}=$ $4 /(3 \times 79), p_{51}^{\prime}=4 / 79, p_{35}^{\prime}=5 /(3 \times 79)$, and $p_{53}^{\prime}=5 /(2 \times$ $79)$.

Proof: Using the fact given in Lemma 4.1, the rest of the proof is similar to that of Lemma 3.4.

\section{A. The Model}

In a cluster of $N$ piconets of a heterogeneous system, we have six types of nodes (Masters), namely 79-hop and 1-slot type, 79-hop and 3-slot type, 79-hop and 5-slot type, 23-hop and 1-slot type, 23-hop and 3-slot type, and 23-hop and 5-slot type. Let $N_{\alpha_{1}}$ be the number of 79-hop and 1-slot type nodes, $N_{\beta_{1}}$ be the number of 79-hop and 3-slot type nodes, $N_{\gamma_{1}}$ be the number of 79-hop and 5-slot type nodes, $N_{\alpha_{2}}$ be the number of 23-hop and 1-slot type nodes, $N_{\beta_{2}}$ be the number of 23-hop and 3-slot type nodes, and $N_{\gamma_{2}}$ be the number of 23-hop and 5-slot type nodes. We have $N=N_{\alpha_{1}}+N_{\beta_{1}}+N_{\gamma_{1}}+N_{\alpha_{2}}+N_{\beta_{2}}+N_{\gamma_{2}}$. We now construct a probabilistic graph $G$ of $N$ nodes in the same way as that stated in the previous section. Also, let $X_{N}$ be the random variable of the number of nodes having no any incident edge. We wanted to develop the model for $E\left(X_{N}\right)$.

Lemma 4.5: Given a probabilistic graph $G$ as defined above, let $p_{\alpha_{1}}$ be the probability that a node of the 79-hop and 1-slot type has no incident edge, $p_{\beta_{1}}$ be the probability that a node of the 79-hop and 3-slot type has no incident edge, $p_{\gamma_{1}}$ be the probability that a node of the 79-hop and 5-slot type has no incident edge, $p_{\alpha_{2}}$ be the probability that a node of the 23-hop and 1-slot type has no incident edge, $p_{\beta_{2}}$ be the probability that a node of the 23-hop and 3-slot type has no incident edge, and $p_{\gamma_{2}}$ be the probability that a node of the 23-hop and 5-slot type has no incident edge. Also, let $p_{i j}$ be the packet interference rates defined in Lemma 3.1, or Lemma 3.2, or Lemma 3.3, or Lemma 3.4 , and let $p_{i j}^{\prime}$ be the packet interference rates defined in Lemma 4.1, or Lemma 4.2, or Lemma 4.3, or Lemma 4.4. Then, we have $p_{\alpha_{1}}=\left(1-p_{11}\right)^{N_{\alpha_{1}}-1} \cdot\left(1-p_{13}\right)^{N_{\beta_{1}}} \cdot(1-$ $\left.p_{15}\right)^{N_{\gamma_{1}}} \cdot\left(1-p_{11}^{\prime}\right)^{N_{\alpha_{2}}} \cdot\left(1-p_{13}^{\prime}\right)^{N_{\beta_{2}}} \cdot\left(1-p_{15}^{\prime}\right)^{N_{\gamma_{2}}}, p_{\beta_{1}}=$

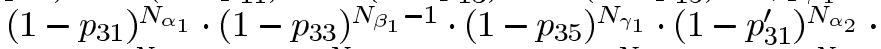

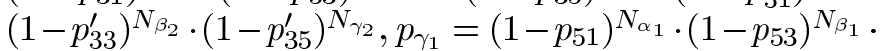

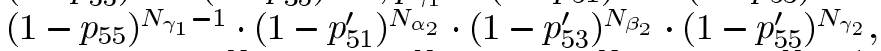

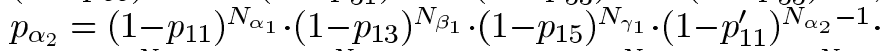

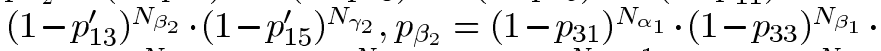

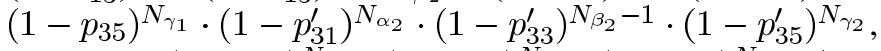
and $p_{\gamma_{2}}=\left(1-p_{51}\right)^{N_{\alpha_{1}}} \cdot\left(1-p_{53}\right)^{N_{\beta_{1}}} \cdot\left(1-p_{55}\right)^{N_{\gamma_{1}}} \cdot(1-$

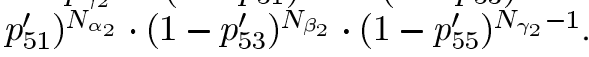

Proof: Similar to that of Lemma 3.5.

Let $E\left(X_{N_{\alpha_{1}}}\right)$ be the expected number of uninterfered 79-hop and 1-slot type nodes, $E\left(X_{N_{\beta_{1}}}\right)$ be the expected number of uninterfered 79-hop and 3-slot type nodes, $E\left(X_{N_{\gamma_{1}}}\right)$ be the expected number of uninterfered 79-hop and 5-slot type nodes, $E\left(X_{N_{\alpha_{2}}}\right)$ be the expected number of uninterfered 23-hop and 1-slot type nodes, $E\left(X_{N_{\beta_{2}}}\right)$ be the expected number of uninterfered 23-hop and 3-slot type nodes, and $E\left(X_{N_{\gamma_{2}}}\right)$ be the expected number of uninterfered 23-hop and 5-slot type nodes, using the fact that $X_{N_{\alpha_{1}}}, X_{N_{\beta_{1}}}, X_{N_{\gamma_{1}}}, X_{N_{\alpha_{2}}}, X_{N_{\beta_{2}}}, X_{N_{\gamma_{2}}}$ all are independent random variables, the following theorem can be easily proved.

Theorem 4.1: Let $G$ be a probabilistic graph with $N_{\alpha 1}$ nodes of 79-hop and 1-slot type, $N_{\beta_{1}}$ nodes of 79-hop and 3-slot type, $N_{\gamma_{1}}$ nodes of 79-hop and 5-slot type, $N_{\alpha_{2}}$ nodes of 23-hop and 1-slot type, $N_{\beta_{2}}$ nodes of 23-hop and 3-slot type, and $N_{\gamma_{2}}$ nodes of 23-hop and 5-slot type, where the probabilities of edges between nodes are specified as in Lemma 3.1 and Lemma 4.1, or Lemma 3.2 and Lemma 4.2, or Lemma 3.3 and Lemma 4.3, or Lemma 3.4 and Lemma 4.4. Also, let $E\left(X_{N}\right)$ be the expected number of nodes of all types having no incident edge, $E\left(X_{N_{\alpha_{1}}}\right)$ be the expected number of nodes of 79-hop and 1-slot type having no incident edge, $E\left(X_{N_{\beta_{1}}}\right)$ be the expected number of nodes of 79-hop and 3-slot type having no incident edge, $E\left(X_{N_{\gamma_{1}}}\right)$ be the expected number of nodes of 79-hop and 5-slot type having no incident edge, $E\left(X_{N_{\alpha_{2}}}\right)$ be the expected number of nodes of 23-hop and 1-slot type having no incident edge, $E\left(X_{N_{\beta_{2}}}\right)$ be the expected number of nodes of 23-hop and 3-slot type having no incident edge, and $E\left(X_{N_{\gamma_{2}}}\right)$ be the expected number of nodes of 23-hop and 5-slot type having no incident edge. We have

$$
\begin{aligned}
& E\left(X_{N_{\alpha_{1}}}\right)=\sum_{r_{\alpha_{1}}=1}^{N_{\alpha_{1}}} r_{\alpha_{1}} \cdot\left(\begin{array}{c}
N_{\alpha_{1}} \\
r_{\alpha_{1}}
\end{array}\right) p_{\alpha_{1}}^{r_{\alpha_{1}}}\left(1-p_{\alpha_{1}}\right)^{N_{\alpha_{1}}-r_{\alpha_{1}}} \\
& E\left(X_{N_{\beta_{1}}}\right)=\sum_{r_{\beta_{1}}=1}^{N_{\beta_{1}}} r_{\beta_{1}} \cdot\left(\begin{array}{c}
N_{\beta_{1}} \\
r_{\beta_{1}}
\end{array}\right) p_{\beta_{1}}^{r_{\beta_{1}}}\left(1-p_{\beta_{1}}\right)^{N_{\beta_{1}}-r_{\beta_{1}}} \\
& E\left(X_{N_{\gamma_{1}}}\right)=\sum_{r_{\gamma_{1}}=1}^{N_{\gamma_{1}}} r_{\gamma_{1}} \cdot\left(\begin{array}{c}
N_{\gamma_{1}} \\
r_{\gamma_{1}}
\end{array}\right) p_{\gamma_{1}}^{r_{\gamma_{1}}}\left(1-p_{\gamma_{1}}\right)^{N_{\gamma_{1}}-r_{\gamma_{1}}} \\
& E\left(X_{N_{\alpha_{2}}}\right)=\sum_{r_{\alpha_{2}}=1}^{N_{\alpha_{2}}} r_{\alpha_{2}} \cdot\left(\begin{array}{c}
N_{\alpha_{2}} \\
r_{\alpha_{2}}
\end{array}\right) p_{\alpha_{2}}^{r_{\alpha_{2}}}\left(1-p_{\alpha_{2}}\right)^{N_{\alpha_{2}}-r_{\alpha_{2}}} \\
& E\left(X_{N_{\beta_{2}}}\right)=\sum_{r_{\beta_{2}}=1}^{N_{\beta_{2}}} r_{\beta_{2}} \cdot\left(\begin{array}{c}
N_{\beta_{2}} \\
r_{\beta_{2}}
\end{array}\right) p_{\beta_{2}}^{r_{\beta_{2}}}\left(1-p_{\beta_{2}}\right)^{N_{\beta_{2}}-r_{\beta_{2}}} \\
& E\left(X_{N_{\gamma_{2}}}\right)=\sum_{r_{\gamma_{2}}=1}^{N_{\gamma_{2}}} r_{\gamma_{2}} \cdot\left(\begin{array}{c}
N_{\gamma_{2}} \\
r_{\gamma_{2}}
\end{array}\right) p_{\gamma_{2}}^{r_{\gamma_{2}}}\left(1-p_{\gamma_{2}}\right)^{N_{\gamma_{2}}-r_{\gamma_{2}}}
\end{aligned}
$$

and

$$
\begin{aligned}
E\left(X_{N}\right)= & E\left(X_{N_{\alpha_{1}}}\right)+E\left(X_{N_{\beta_{1}}}\right)+E\left(X_{N_{\gamma_{1}}}\right) \\
& +E\left(X_{N_{\alpha_{2}}}\right)+E\left(X_{N_{\beta_{2}}}\right)+E\left(X_{N_{\gamma_{2}}}\right)
\end{aligned}
$$

where $p_{\alpha_{1}}, p_{\beta_{1}}, p_{\gamma_{1}} p_{\alpha_{2}}, p_{\beta_{2}}$, and $p_{\gamma_{2}}$ are defined in Lemma 4.5.

Using Theorem 4.1, the aggregated throughput of a heterogeneous system under symmetric traffic can be given by the following expression:

$$
\begin{aligned}
& \text { Aggregated throughput } \\
& \begin{aligned}
= & E\left(X_{N_{\alpha_{1}}}\right) \times 10^{6} \times(240 / 625) \\
& +E\left(X_{N_{\beta_{1}}}\right) \times 10^{6} \times(1464 / 1875) \\
& +E\left(X_{N_{\gamma_{1}}}\right) \times 10^{6} \times(2712 / 3125) \\
& +E\left(X_{N_{\alpha_{2}}}\right) \times 10^{6} \times(240 / 625) \\
& +E\left(X_{N_{\beta_{2}}}\right) \times 10^{6} \times(1464 / 1875) \\
& +E\left(X_{N_{\gamma_{2}}}\right) \times 10^{6} \times(2712 / 3125) \text { bits/s. }
\end{aligned}
\end{aligned}
$$




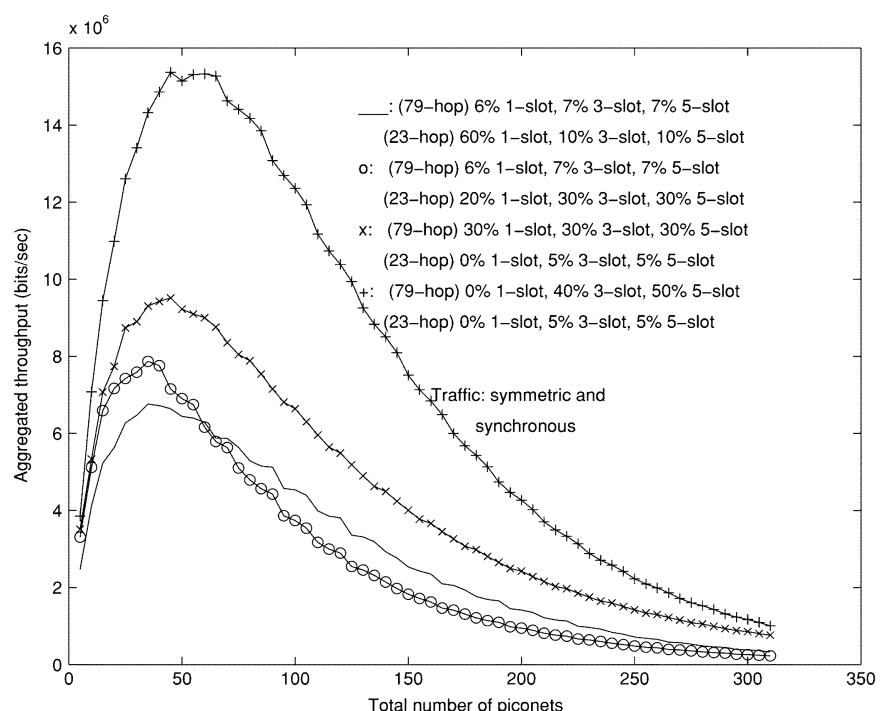

Fig. 25. Aggregated throughputs of various symmetric and synchronous traffic.

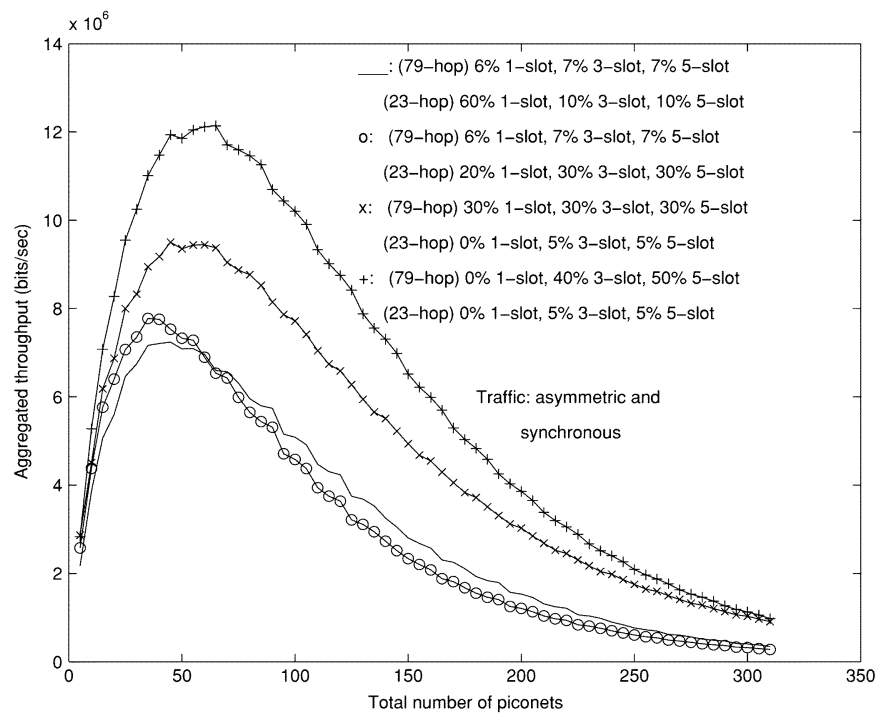

Fig. 26. Aggregated throughputs of various asymmetric and synchronous traffic.

Also, the aggregated throughput of a heterogeneous system under asymmetric traffic can be given by the following expression:

\section{Aggregated throughput}

$$
\begin{aligned}
= & E\left(X_{N_{\alpha_{1}}}\right) \times 10^{6} \times(240 / 625) \\
& +E\left(X_{N_{\beta_{1}}}\right) \times 10^{6} \times((1464 / 1875)+(240 / 625)) / 2 \\
& +E\left(X_{{N_{1}}_{1}}\right) \times 10^{6} \times((2712 / 3125)+(240 / 625)) / 2 \\
& +E\left(X_{N_{\alpha_{2}}}\right) \times 10^{6} \times(240 / 625) \\
& +E\left(X_{N_{\beta_{2}}}\right) \times 10^{6} \times((1464 / 1875)+(240 / 625)) / 2 \\
& +E\left(X_{N_{\gamma_{2}}}\right) \times 10^{6} \times((2712 / 3125) \\
& +(240 / 625)) / 2 \text { bits/s. }
\end{aligned}
$$

The aggregated throughputs of various combinations of different types of piconets under different traffic conditions are shown in Figs. 25-28. These plots suggest the following.

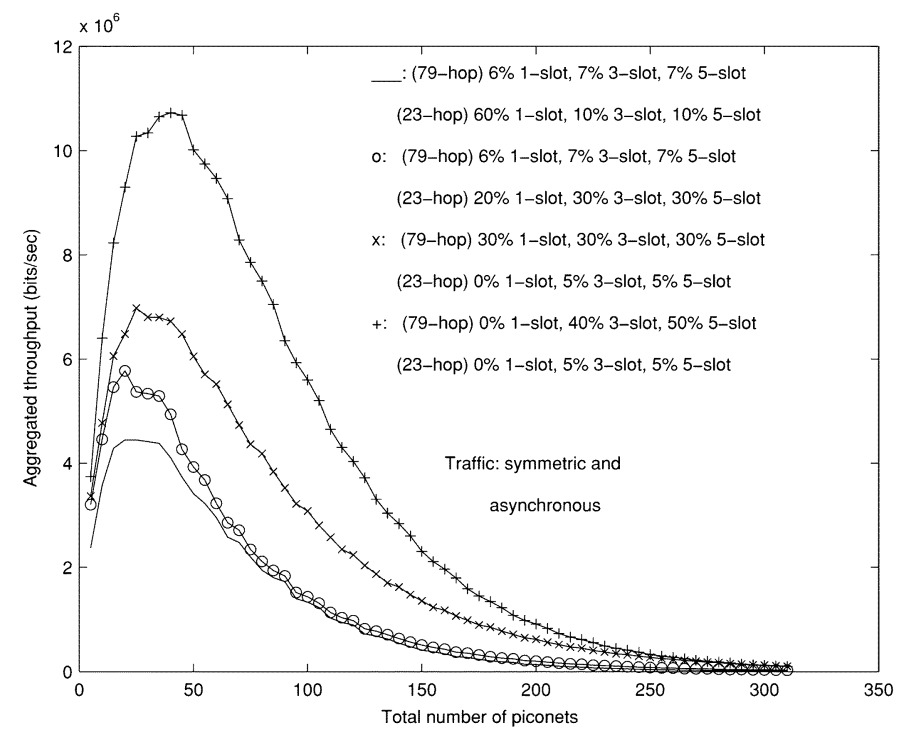

Fig. 27. Aggregated throughputs of various symmetric asynchronous traffic.

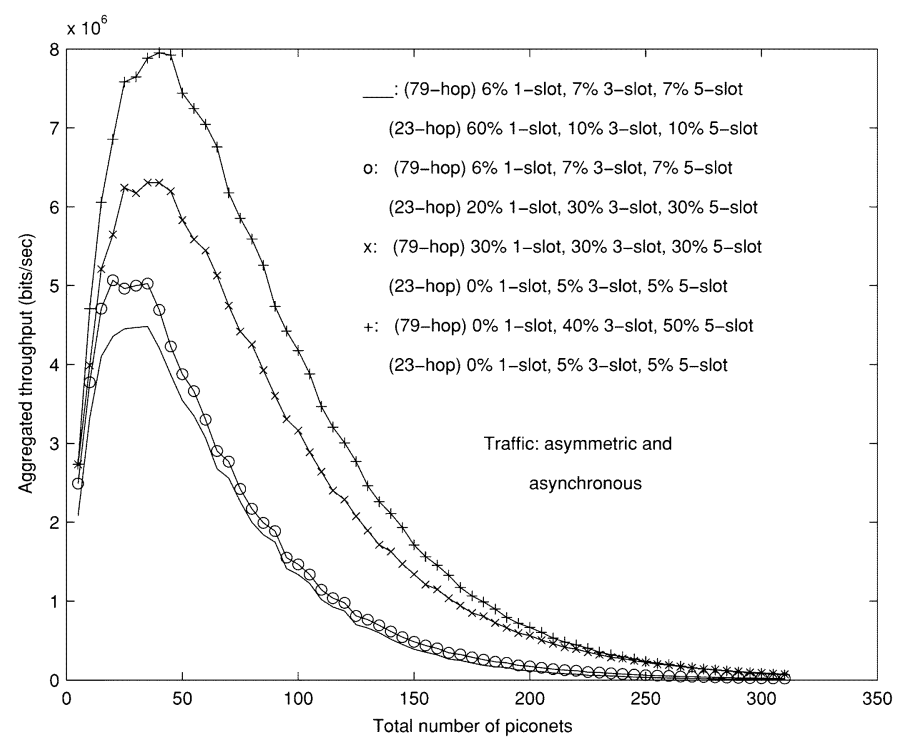

Fig. 28. Aggregated throughputs of various asymmetric and asynchronous traffic.

- If all the piconets operate synchronously, in both symmetric and asymmetric modes, it produces higher aggregated throughput, compared with the unsynchronized system. The synchronous system also allows us to accommodate more piconets, say between 40 and 50 depending on the percentage of 23-hop type piconets in the cluster, in an area to reach the maximum aggregated throughput, compared with the unsynchronized one, which can accommodate less piconets in general to reach its maximum aggregated throughput.

- Whether the piconets operate synchronously or asynchronously and despite the percentage of 23-hop type of piconets, in general, the system with symmetric traffic gain higher aggregated throughput than that gained by the one with asymmetric traffic. This is not hard to predict since 
twice of the one-way symmetric data rate is more than the sum of the upstream and down stream data rate for asymmetric traffic in a channel.

- Under any kind of traffic conditions, the percentage of 23-hop type piconets in the cluster affects the maximum aggregated throughput and the number of piconets the cluster can accommodate to reach the maximum throughput. In general, the higher the percentage of the 23-hop type is, the lower the maximum aggregated throughput will be, and the smaller the number of piconets the cluster can accommodate.

- We use Fig. 28 to conclude the discussion on the model of heterogeneous system. When forming a cluster of piconets in an area using a given number of 23-hop and 79-hop types Bluetooth devices, if the given number of devices is less than $(1+7) \times 30=240$, try to form the cluster in such a way that the number of piconets is close to 30 . In case the number of Bluetooth devices in the area is more than 240 , try to form the cluster of piconets in the way that we have as smaller number of piconets as possible. In general, it is not a good idea to accommodate more than 30 piconets in a cluster of heterogeneous piconets.

\section{CONCLUSION}

We developed a model of packet interference in a cluster of homogeneous piconets and a model of packet interference in a cluster of heterogeneous piconets using multiple-slot packets. We show how the aggregate throughput in a cluster of piconets degrade under various traffic scenarios, such as 1-slot, 3-slot, and 5-slot packets in symmetric and asymmetric modes in synchronous and asynchronous conditions of Master clocks. Our analytic model is developed based on the idea of probabilistic graphs, where a node denotes a piconet and an edge denotes the probability of interference between two nodes. The concept of probabilistic graphs used in modeling packet interference in a collection of Bluetooth piconets gives us several advantages. First, we can easily model packet interference in a heterogeneous collection of piconets by suitably defining edge weights which represent packet interference between two nodes. Second, it is easy to extend this idea to asynchronous and synchronous traffic involving multislot packets. Finally, once a probabilistic graph is constructed, computation of interference rates is a matter of computing the properties of the graph, such as size of average clique. In addition, our model can be employed to help form the optimal (or near-optimal) configuration of a cluster of piconets to gain maximum (or close to maximum) aggregated throughput.

\section{REFERENCES}

[1] A. El-Hoiydi, "Interference between Bluetooth networks-upper bound on the packet error rate," IEEE Commun. Lett., vol. 5, no. 6, pp. 245-247, Jun. 2001.
[2] G. Ennis, "Impact of Bluetooth on 802.11 direct sequence," IEEE 802.11-98/319, Sep. 2001.

[3] N. Golmie, "Bluetooth and 802.11 interference: Simulation model and system results," IEEE 802.15-01/195RO, Apr. 2001.

[4] The Bluetooth Special Interest Group. Baseband Specification Version 1.1 (2001). [Online]. Available: http://www.bluetooth.com

[5] J. Lansford, A. Stephens, and R. Nevo, "Wi-fi (802.11b) and Bluetooth: Enabling coexistence," IEEE Netw., pp. 20-27, Sep./Oct. 2001.

[6] V. Mitter, I. Howitt, and J. Gutierrez, "Empirical study for ieee 802.11b WLAN and Bluetooth coexistence in UL band," IEEE 802.15-01/148TG2, Mar. 2001.

[7] K. Naik, D. S. L. Wei, and Y. T. Su, "Packet interference in a heterogeneous cluster of Bluetooth piconets," in IEEE Veh. Technol. Conf., Oct. 2003, pp. 582-586.

[8] —_ "Analysis of packet interference in a cluster of Bluetooth piconets under different traffic conditions," in Proc. IEEE Int. Conf. Commun. (ICC), Paris, France, Junel 2004, pp. 3450-3454.

[9] S. Shellhammer, "Packet error rate of an IEEE 802.11 WLAN in the presence of Bluetooth," IEEE P802.15-00/133rO, May 2000.

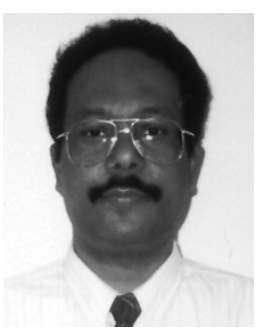

K. Naik (M'94) received the B.S. degree from Sambalpur University, Sambalpur, India, the M.Tech. degree from the Indian Institute of Technology, Kharagpur, India, the M.Math. degree in computer science from the University of Waterloo, Waterloo, ON, Canada, and the Ph.D. degree in electrical and computer engineering from Concordia University, Montreal, QC, Canada.

He was a Faculty Member at the University of Aizu, Aizu, Japan, and Carleton University, Ottawa, ON, Canada. At present, he is an Associate Professor in the Department of Electrical and Computer Engineering, University of Waterloo. He was a Program Co-Chair of the 5th International Conference on Information Technology, Bhubaneswar, India, December 2002. He is on the program committee of several international conferences in the area of wireless communication and mobile computing. His research interests are testing of communication protocols, wireless communication, resource allocation in cellular and 3G systems, sensor networks, ad hoc networks, MAC protocols for wireless LAN, Bluetooth networks, mobile computing, and peer-to-peer communication.

Dr. Naik is a Co-Guest Editor of the IEEE JOURNAL ON SELECTED AREAS IN COMMUNICATIONS for the Special Issue on Mobile Computing and Networking.

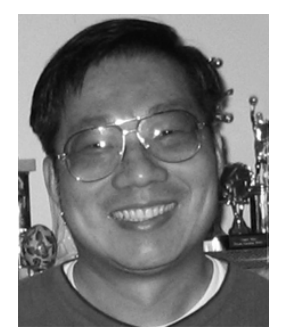

David S. L. Wei (S'87-M'94) received the Ph.D. degree in computer and information science from the University of Pennsylvania, Philadelphia, in 1991.

$\mathrm{He}$ is currently an Associate Professor of Computer and Information Science at Fordham University, Bronx, NY. From May 1993 to August 1997, he was on the Faculty of the School of Computer Science and Engineering, University of Aizu, Aizu, Japan, as an Associate Professor and then a Professor. He has authored and coauthored more than 70 technical papers in the areas of distributed and parallel processing, wireless networks and mobile computing, and optical networks in various archival journals and conference proceedings. $\mathrm{He}$ served on the program committee and was a session chair for several reputed international conferences. He served as Co-Chair of Power Aware Communication and Software, Minitrack in the Software Track at the 34th Hawaii International Conference on Systems Sciences (HICSS-34). He focuses his research effort on wireless networks, mobile computing, and peer-to-peer communications.

Dr. Wei is a Lead Guest Editor of the IEEE JouRnAL ON SELECTED AREAS IN COMMUNICATIONS for the Special Issue on Mobile Computing and Networking. 


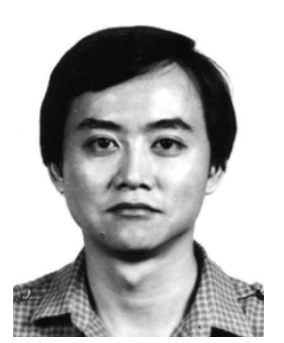

Yu T. Su (S'81-M'83) received the Ph.D. degree in electrical engineering from the University of Southern California, Los Angeles, in 1983.

From 1983 to 1989, he was with LinCom Corporation, Los Angeles, CA, where he was a Corporate Scientist and was involved in the design of various measurement and digital satellite communication systems. Since September 1989, he has been with the National Chiao Tung University, Hsinchu, Taiwan, where he was head of the Communication Engineering Department between 2001 and 2003. He is also affiliated with the Microelectronics and Information Systems Research Center, National Chiao Tung University and served as a Deputy Director from 1997 to 2000. His main research interests include communication theory and statistical signal processing.

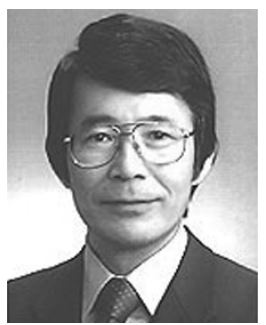

Norio Shiratori (M'87-SM'96-F'98) received the M.E. and Ph.D. degrees in electrical and communication engineering from Tohoku University, Sendai, Japan, in 1974 and 1977, respectively.

Since 1977, he has been with the Research Institute of Electrical Communication (RIEC), Tohoku University. He is a Professor of Computer Science at RIEC and also the Vice Director of the department since April 2004. His research has been in the area of computer networks and worked on performance evaluation of computer networks, and focused on protocol specification environment. He has also worked on communication software and human interface. He has proposed a new concept of flexible computing and is still working in this direction. His recent research interest is in ubiquitous and symbiosis computing.

Dr. Shiratori is a Fellow of the Institute of Electrical, Information, and Communication Engineers (IEICE) and IPSJ. 